\title{
Photoinduced Electron Transfer Within Supramolecular Donor-Acceptor Peptide Nanostructures Under Aqueous Conditions
}

Allix M. Sanders, ${ }^{1}$ Timothy J. Magnanelli, ${ }^{1}$ Arthur E. Bragg, ${ }^{1}$ and John D. Tovar ${ }^{1,2,3 *}$

1. Department of Chemistry, Krieger School of Arts and Sciences, 2. Department of Materials Science and Engineering, Whiting School of Engineering, 3. Institute of NanoBioTechnology, Johns Hopkins University, 3400 N. Charles St., Baltimore, MD 21218 USA

\section{Supporting Information}

\section{Table of Contents}

Experimental details and characterization data

NMR spectra

ESI mass spectra

HPLC traces of purified peptides

Additional TEM images

Additional circular dichroism spectra

Additional ultrafast spectra and characterization 


\section{Experimental Details and Characterization Data}

General considerations: DMF was purchased from Sigma-Aldrich and dried over $4 \AA$ molecular sieves. Solvents were degassed by sparging with nitrogen for 30 to 90 minutes before use. Tetrakis(triphenylphosphine)palladium was obtained from Strem Chemicals. Wang-Lys(Mtt) was purchased from Chem-Impex International. N-Methylpyrrolidone (NMP), Wang-Val resin, Wang-Lys resin, and Fmoc-protected amino acids were obtained from Advanced ChemTech. $O$ (Benzotriazol-1-yl)- $N, N, N^{\prime}, N^{\prime}$-tetramethyluronium hexafluorophosphate (HBTU) was purchased from Oakwood Products Inc. All other reagents and starting materials were obtained from SigmaAldrich and were used as received. The side-chain amine of Wang-Lys resin was acylated via treatment with acetic anhydride. 5,5'-bis-tributylstannyl-[2,2']-bithiophene, and 5bromothiophene-2-carboxylic acid were prepared using literature procedures. ${ }^{1,2}$

NMR Spectroscopy: ${ }^{1} \mathrm{H}-\mathrm{NMR}$ spectra were obtained using a Bruker Avance $400 \mathrm{MHz}$ FT-NMR spectrometer and processed with Bruker Topspin 1.3. Peptide ${ }^{1} \mathrm{H}$ NMR spectra were acquired using a 1 second presaturation pulse to suppress water.

Electrospray Ionization Mass Spectrometry (ESI-MS): ESI samples were collected using a Thermo Finnigan LCQ Deca Ion Trap Mass Spectrometer in negative mode. Samples were prepared in a 1:1 MeOH:water solution with $0.1 \%$ ammonium hydroxide.

UV-Vis and Photoluminescence: UV-Vis spectra were obtained using a Varian Cary 50 Bio UVVis spectrophotometer. Photoluminescence spectra were obtained using a PTi Photon Technology International Fluorometer with an Ushio Xenon short arc lamp. Spectroscopic samples were 
prepared at $2.2 \mu \mathrm{M}$ in Millipore water. The $\mathrm{pH}$ was then adjusted by adding $10 \mu \mathrm{L}$ of either $1 \mathrm{M}$ $\mathrm{KOH}$ (basic) or $1 \mathrm{M} \mathrm{HCl} \mathrm{(acidic).}$

Circular Dichroism (CD): CD spectra were obtained using a Jasco J-810 spectropolarimeter. Spectroscopic samples were prepared at $4.4 \mu \mathrm{M}$ in Millipore water. The $\mathrm{pH}$ was then adjusted by adding $10 \mu \mathrm{L}$ of either $1 \mathrm{M} \mathrm{KOH}$ (basic) or $1 \mathrm{M} \mathrm{HCl}$ (acidic).

Reverse-Phase HPLC: HPLC purification was performed on a Varian PrepStar SD-1 (preparative) instrument using Luna $5 \mu \mathrm{m}$ particle diameter C8 with TMS endcapping columns with silica solid support. An ammonium formate aqueous buffer $(\mathrm{pH} 8)$ and acetonitrile were used as the mobile phase.

Transmission Electron Microscopy (TEM): Imaging was performed on a Philips EM 420 transmission electron microscope equipped with an SIS Megaview III CCD digital camera. The samples were prepared by pipetting a drop of $1 \mathrm{mg} / \mathrm{mL}$ solution of assembled peptide in water onto 200 mesh Formvar coated copper grids and incubated for 5 minutes at $25^{\circ} \mathrm{C}$. Excess solution was wicked off by touching the side of the grid to filter paper. The sample was then stained with a $2 \%$ uranyl acetate solution, immediately washed by dipping in water, and excess moisture was wicked off. The grid was allowed to dry in air before imaging.

Ultrafast Transient Absorption (TA) Spectroscopy: Ultrafast spectroscopic characterization was accomplished using an amplified Ti:Sapphire system as described in previous works. ${ }^{3,4}$ Ultrafast laser pulses ( 35-fs duration, 800-nm center wavelength) were generated with an amplified Ti:sapphire laser system (Coherent Legend Elite), producing approximately $4.0 \mathrm{~W}$ at a repetition rate of $990 \mathrm{~Hz}$. A small fraction of the beam $(<1 \mathrm{~mW})$ was focused into a sapphire crystal 
to generate a broadband white-light continuum covering a range of $450-1150 \mathrm{~nm}$. Another fraction of the amplifier output was passed through a Type I BBO, SHG crystal or used to pump a femtosecond optical parametric amplifier (Coherent, OPerA Solo) to generate $400 \mathrm{~nm}$ and $480 \mathrm{~nm}$ photoexcitation pulses, respectively. Optical delay of the photoexcitation pulse relative to the probe continuum was controlled with a translation stage (Newport). Excitation pulses were focused to diameters of $0.5-1.0 \mathrm{~mm}$ that ensured complete coverage of the white light spot ( 100 micron) and prevented effects due to spatial chirp. The dispersed spectrum of the probe beam was collected after the sample using an Acton SP 2360 spectrograph outfitted with a Pixis 100BR CCD-array detector.

Each set of spectra was collected in two parts, $450-800$ and 800-1150 nm, with each nearinfrared measurement conducted immediately after the visible measurement without altering sample position or alignment conditions. All samples were studied at the same concentration unless otherwise indicated. Assembled samples were stirred to maintain a homogeneous distribution of aggregates. Collected spectra were smoothed, chirp-corrected, and combined to make the sets plotted in Figures $7 \mathrm{a}$ and $7 \mathrm{~b}$. Time zero was uniformly set to the point at which the primary peak reached $50 \%$ of its maximum intensity. The time resolution given by the half-rise of the instrument response was found to be 90 fs for measurements using 400 and $480 \mathrm{~nm}$ excitation pulses. None of the samples suffered degradation due to laser exposure or storage over the timeframe of the conducted measurements.

$N$-propyl-1,4,5,8-naphthalenetetracarboxylic acid monoanhydride: The synthesis of this molecule was adapted from a prior report. ${ }^{5}$ 1,4,5,8-Naphthalenetetracarboxylic acid dianhydride (10.7 g, $40.0 \mathrm{mmol})$ was added to a three neck round bottomed flask equipped with a condenser and dissolved in DMF (300 mL). The mixture was heated to $140^{\circ} \mathrm{C}$. Propylamine (3.28 $\mathrm{mL}, 40.0$ 
mmol), was diluted in DMF (25 mL) and added dropwise over 2.5 hours to the reaction flask via syringe pump. The mixture was further heated to reflux for 15 hours, then allowed to return to room temperature. $500 \mathrm{~mL}$ of a saturated $\mathrm{NH}_{4} \mathrm{Cl}$ aqueous solution was added. The light brown solid was collected by filtration, then washed with water. The crude solid was suspended in boiling chloroform, and the suspension was filtered. The solvent was removed from the mother liquor to give the desired product as an off-white solid (5.26 g, $17.0 \mathrm{mmol}, 43 \%)$ whose characterization data matched the literature and that was used without further purification. ${ }^{1} \mathrm{H} \mathrm{NMR}(400 \mathrm{MHz}$, $\left.\mathrm{CDCl}_{3}\right) \delta: 8.82(\mathrm{~s}, 4 \mathrm{H}), 4.21-4.15(\mathrm{~m}, 2 \mathrm{H}, J), 1.78(\mathrm{sext}, 2 \mathrm{H}, J=7.6 \mathrm{~Hz}), 1.03(\mathrm{t}, 3 \mathrm{H}, J=7.4 \mathrm{~Hz})$. ${ }^{13} \mathrm{C}$ NMR $\left(100 \mathrm{MHz}, \mathrm{CDCl}_{3}\right) \delta: 162.4,159.0,133.3,131.4,129.0,128.1,122.9,42.8,21.5,11.6$.

General Solid Phase Peptide Synthesis (SPPS), $N$-terminus-acylation, and on-resin Stille coupling procedure: Peptides were synthesized via standard SPPS using Fmoc-protected amino acids, starting from Wang resin preloaded with the first amino acid (Wang-Val $=0.7$ or 0.4 $\mathrm{mmol} / \mathrm{g}$, Wang-Lys $(\mathrm{Mtt})=0.47 \mathrm{mmol} / \mathrm{g}$, Wang-Lys $(\mathrm{Ac})=0.37 \mathrm{mmol} / \mathrm{g})$, acylated with 5 bromothiophene-2-carboxylic acid and subjected to Stille cross-coupling conditions in the presence of 5,5'-bis-tributylstannyl-[2,2']-bithiophene as previously described. ${ }^{1}$

General On-Resin Deprotection and Imidation Procedure: Following SPPS, $N$-terminusacylation, and On-Resin Stille coupling procedures, the resin was dried and placed in a Schlenk tube equipped with a reflux condenser. $N$-propyl-1,4,5,8-naphthalenetetracarboxylic acid monoanhydride (3 eq.) and DMF (10 mL) was added and the mixture was heated to $50^{\circ} \mathrm{C}$ for 1 hour, with continuous $\mathrm{N}_{2}$ bubbling through the solution. The suspension was then heated to $110^{\circ} \mathrm{C}$ for 22 hours. The mixture was allowed to cool, then the resin was transfer to a peptide chamber and subjected to a wash cycle (3x NMP, 3x DMF, 2x iPrOH, 2x $\mathrm{H}_{2} \mathrm{O}, 2 \mathrm{x}(2 \mathrm{x}$ THF, $2 \mathrm{x}$ iPrOH), 2x acetonitrile, $2 \mathrm{x}$ diethylether, $2 \mathrm{x}$ hexanes). 
General cleavage, work-up procedure of peptides: Following solid-phase cross-coupling and imidation (if applicable) peptide was cleaved from the resin with a 95\% TFA cocktail, isolated, and HPLC purified as previously described. ${ }^{1}$

HO-K(NDI)VEVGG-OT4-GGVEVK(NDI)-OH peptide (DA-6): Solid supported WangK(Mtt)VEVGG-NH2 peptide $N$-acylated with 5-bromothiophene-2-carboxylic acid was prepared $(0.46 \mathrm{mmol})$ and subjected to the standard Stille coupling procedure in the presence of 5,5'-bistributylstannyl-[2,2']-bithiophene $(0.23 \mathrm{mmol}, 0.17 \mathrm{~g})$ and $\mathrm{Pd}\left(\mathrm{PPh}_{3}\right)_{4}(0.018 \mathrm{mmol}, 0.021 \mathrm{~g})$ for 19 hours. The lysine residues of the resulting solid-supported peptide were then subjected to the deprotection and imidation procedure with $N$-propyl-1,4,5,8-naphthalenetetracarboxylic acid monoanhydride (1.4 mmol, $0.43 \mathrm{~g}$ ) for 22 hours. Following general cleavage, work-up, and HPLC purification, the peptide was obtained as a light orange powder $(0.0032 \mathrm{mmol}, 0.0063 \mathrm{~g}, 1.3 \%$ yield). UV-Vis $\left(\mathrm{H}_{2} \mathrm{O}\right) \lambda / \mathrm{nm}(\log \varepsilon): 386$ (4.32). MS (ESI) $m / z 1068.8(\mathrm{M}-2 \mathrm{H})^{-2}$ (calc. 1068.8), $m / z 712.3(\mathrm{M}-3 \mathrm{H})^{-3}$ (calc. 712.2), $m / z 534.2(\mathrm{M}-3 \mathrm{H})^{-3}$ (calc. 533.9).

HO-VEVK(NDI)GG-OT4-GGK(NDI)VEV-OH peptide (DA-3): Solid supported WangVEVK(Mtt)GG-NH 2 peptide $N$-acylated with 5-bromothiophene-2-carboxylic acid was prepared $(0.50 \mathrm{mmol})$ and subjected to the standard Stille coupling procedure in the presence of 5,5'-bistributylstannyl-[2,2']-bithiophene $(0.25 \mathrm{mmol}, 0.19 \mathrm{~g})$ and $\mathrm{Pd}\left(\mathrm{PPh}_{3}\right)_{4}(0.020 \mathrm{mmol}, 0.023 \mathrm{~g})$ for 18 hours. The lysine residues of the resulting solid-supported peptide were then subjected to the deprotection and imidation procedure with $N$-propyl-1,4,5,8-naphthalenetetracarboxylic acid monoanhydride (1.5 mmol, $0.46 \mathrm{~g})$ for 22 hours. Following general cleavage, work-up, and HPLC purification, the peptide was obtained as an orange powder $(0.0068 \mathrm{mmol}, 0.014 \mathrm{~g}, 2.7 \%$ yield $)$. UV-Vis $\left(\mathrm{H}_{2} \mathrm{O}\right) \lambda / \mathrm{nm}(\log \varepsilon): 388$ (4.54). MS (ESI) $m / z$ 1068.8 (M-2H) ${ }^{-2}$ (calc. 1068.9), $m / z 712.3$ $(\mathrm{M}-3 \mathrm{H})^{-3}$ (calc. 712.4), $m / z 534.1(\mathrm{M}-3 \mathrm{H})^{-3}$ (calc. 533.9). 
HO-VEVGK(NDI)G-OT4-GK(NDI)GVEV-OH peptide (DA-2): Solid supported WangVEVK(Mtt)GG-NH 2 peptide $N$-acylated with 5-bromothiophene-2-carboxylic acid was prepared $(0.50 \mathrm{mmol})$ and subjected to the standard Stille coupling procedure in the presence of 5,5'-bistributylstannyl-[2,2']-bithiophene $(0.25 \mathrm{mmol}, 0.19 \mathrm{~g})$ and $\mathrm{Pd}\left(\mathrm{PPh}_{3}\right)_{4}(0.020 \mathrm{mmol}, 0.023 \mathrm{~g})$ for 18 hours. The lysine residues of the resulting solid-supported peptide were then subjected to the deprotection and imidation procedure with $N$-propyl-1,4,5,8-naphthalenetetracarboxylic acid monoanhydride ( $1.5 \mathrm{mmol}, 0.46 \mathrm{~g}$ ) for 22 hours. Following general cleavage, work-up, and HPLC purification, the peptide was obtained as an orange-brown powder $(0.010 \mathrm{mmol}, 0.022 \mathrm{~g}, 4.1 \%$ yield). UV-Vis $\left(\mathrm{H}_{2} \mathrm{O}\right) \lambda / \mathrm{nm}(\log \varepsilon): 387$ (4.60). MS (ESI) $m / z 1068.9(\mathrm{M}-2 \mathrm{H})^{-2}$ (calc. 1068.8), $m / z 712.4(\mathrm{M}-3 \mathrm{H})^{-3}$ (calc. 712.2), $m / z$ 534.1 (M-3H) ${ }^{-3}$ (calc. 533.9).

HO-K(Ac)VEVGG-OT4-GGVEVK(Ac)-OH peptide (C-6): Solid supported WangK(Ac)VEVGG-NH 2 peptide $N$-acylated with 5-bromothiophene-2-carboxylic acid was prepared $(0.43 \mathrm{mmol})$ and subjected to the standard Stille coupling procedure in the presence of 5,5'-bistributylstannyl-[2,2']-bithiophene $(0.22 \mathrm{mmol}, 0.16 \mathrm{~g})$ and $\mathrm{Pd}\left(\mathrm{PPh}_{3}\right)_{4}(0.017 \mathrm{mmol}, 0.020 \mathrm{~g})$ for 20 hours. Following general cleavage, work-up, and HPLC purification, the peptide was obtained as a light orange powder $(0.0075 \mathrm{mmol}, 0.012 \mathrm{~g}, 3.5 \%$ yield $)$. UV-Vis $\left(\mathrm{H}_{2} \mathrm{O}\right) \lambda / \mathrm{nm}(\log \varepsilon): 420$ (4.48). MS (ESI) $m / z 819.7(\mathrm{M}-2 \mathrm{H})^{-2}$ (calc. 819.3), $\mathrm{m} / z 546.2(\mathrm{M}-3 \mathrm{H})^{-3}$ (calc. 545.9), $\mathrm{m} / z 409.5$ $(\mathrm{M}-3 \mathrm{H})^{-3}$ (calc. 409.1).

HO-VEVK(Ac)GG-OT4-GGK(Ac)VEV-OH peptide (C-3): Solid supported WangVEVK(Ac)GG-NH 2 peptide $N$-acylated with 5-bromothiophene-2-carboxylic acid was prepared $(0.5 \mathrm{mmol})$ and subjected to the standard Stille coupling procedure in the presence of 5,5'-bistributylstannyl-[2,2']-bithiophene $(0.25 \mathrm{mmol}, 0.19 \mathrm{~g})$ and $\mathrm{Pd}\left(\mathrm{PPh}_{3}\right)_{4}(0.020 \mathrm{mmol}, 0.023 \mathrm{~g})$ for 20 hours. Following general cleavage, work-up, and HPLC purification, the peptide was obtained 
as a light orange powder $(0.018 \mathrm{mmol}, 0.029 \mathrm{~g}, 7.1 \%$ yield $)$. UV-Vis $\left(\mathrm{H}_{2} \mathrm{O}\right) \lambda / \mathrm{nm}(\log \varepsilon): 417$ (4.59). MS (ESI) $m / z 819.7(\mathrm{M}-2 \mathrm{H})^{-2}$ (calc. 819.3), $m / z 546.2(\mathrm{M}-3 \mathrm{H})^{-3}$ (calc. 545.9), $m / z 409.5$ $(\mathrm{M}-3 \mathrm{H})^{-3}$ (calc. 409.1).

HO-VEVGK(Ac)G-OT4-GK(Ac)GVEV-OH peptide (C-2): Solid supported WangVEVGK(Ac)G-NH $\mathrm{NH}_{2}$ peptide $N$-acylated with 5-bromothiophene-2-carboxylic acid was prepared $(0.5 \mathrm{mmol})$ and subjected to the standard Stille coupling procedure in the presence of 5,5'-bistributylstannyl-[2,2']-bithiophene $(0.25 \mathrm{mmol}, 0.19 \mathrm{~g})$ and $\mathrm{Pd}\left(\mathrm{PPh}_{3}\right)_{4}(0.020 \mathrm{mmol}, 0.023 \mathrm{~g})$ for 20 hours. Following general cleavage, work-up, and HPLC purification, the peptide was obtained as a light orange powder $(0.010 \mathrm{mmol}, 0.016 \mathrm{~g}, 3.9 \%$ yield $)$. UV-Vis $\left(\mathrm{H}_{2} \mathrm{O}\right) \lambda / \mathrm{nm}(\log \varepsilon): 420$ (4.61). MS (ESI) $m / z 819.8(\mathrm{M}-2 \mathrm{H})^{-2}$ (calc. 819.3), $\mathrm{m} / z$ 546.2 (M-3H) ${ }^{-3}$ (calc. 545.9), $\mathrm{m} / z 409.5$ $(\mathrm{M}-3 \mathrm{H})^{-3}$ (calc. 409.1). 


\section{NMR Spectra}

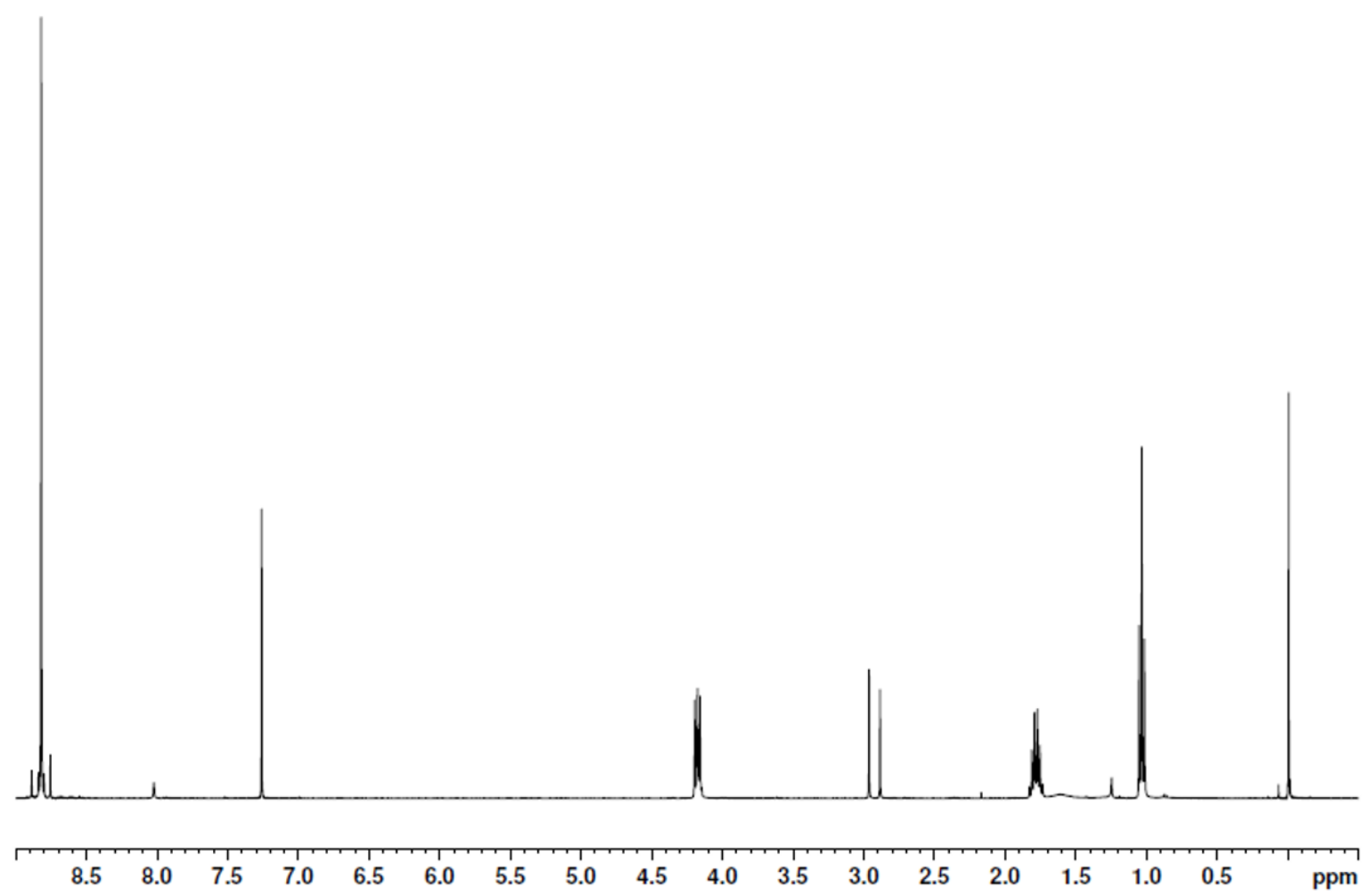

Figure S1. ${ }^{1} \mathrm{H}$ NMR of N-propyl-1,4,5,8-naphthalenetetracarboxylic acid monoanhydride in $\mathrm{CDCl}_{3}$.

${ }^{1} \mathrm{H}$ NMR (400 MHz, $\left.\mathrm{CDCl}_{3}\right) \delta: 8.82(\mathrm{~s}, 4 \mathrm{H}), 4.21-4.15(\mathrm{~m}, 2 \mathrm{H}, J), 1.78$ (sext, 2H, $J=7.6 \mathrm{~Hz}$ ), $1.03(\mathrm{t}, 3 \mathrm{H}, J=7.4 \mathrm{~Hz})$. 


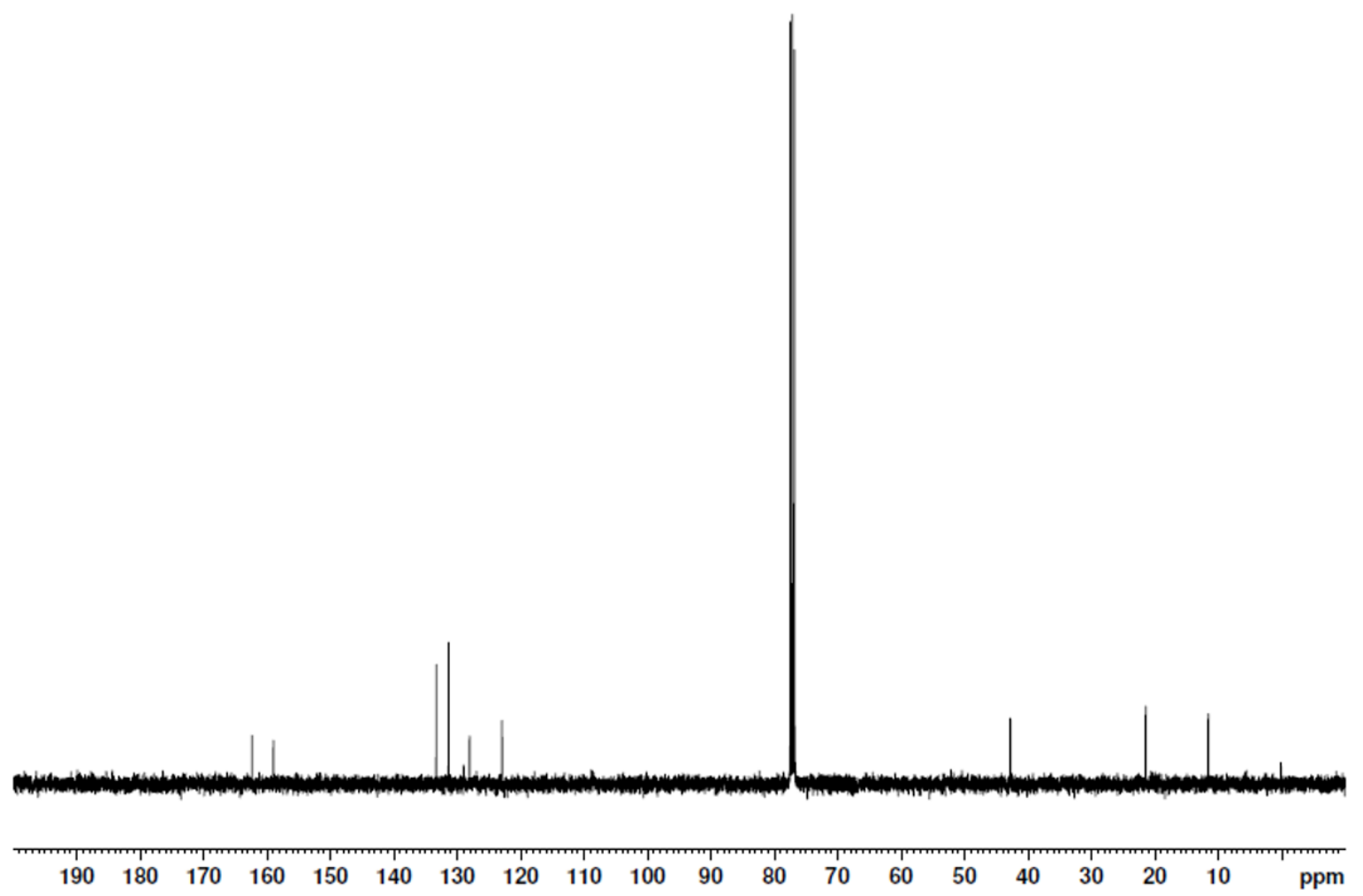

Figure S2. ${ }^{13} \mathrm{C}$ NMR of N-propyl-1,4,5,8-naphthalenetetracarboxylic acid monoanhydride in $\mathrm{CDCl}_{3}$.

${ }^{13} \mathrm{C}$ NMR $\left(100 \mathrm{MHz}, \mathrm{CDCl}_{3}\right) \delta: 162.4,159.0,133.3,131.4,129.0,128.1,122.9,42.8,21.5,11.6$. 


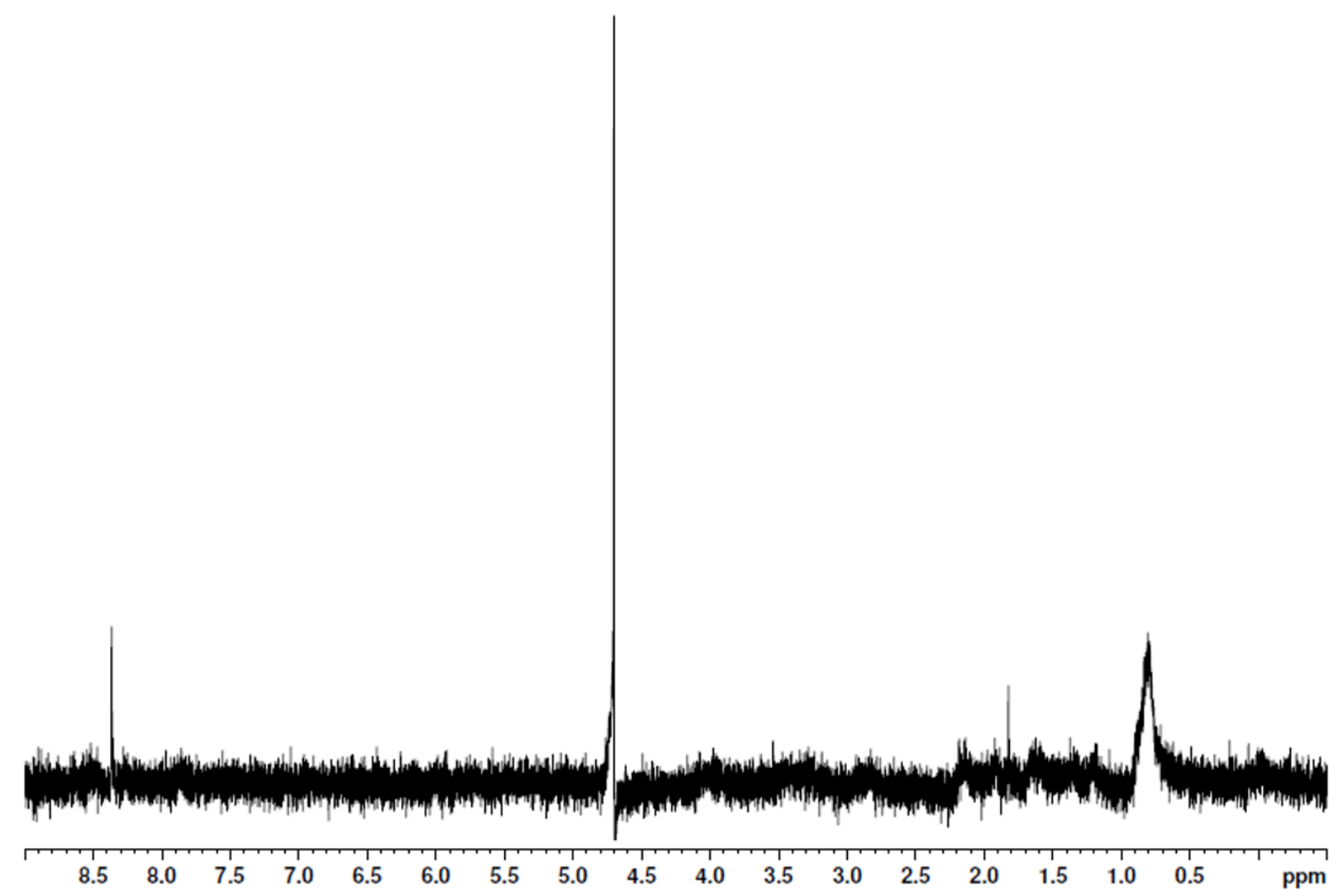

Figure S3. ${ }^{1} \mathrm{H}$ NMR of HO-K(NDI)VEVGG-OT4-GGVEVK(NDI)-OH peptide (DA-6) in $\mathrm{D}_{2} \mathrm{O}$.

Spectrum was acquired using a 1 second presaturation pulse to suppress water. Peak broadness presumably due to aggregation of the peptide during the experiment. 


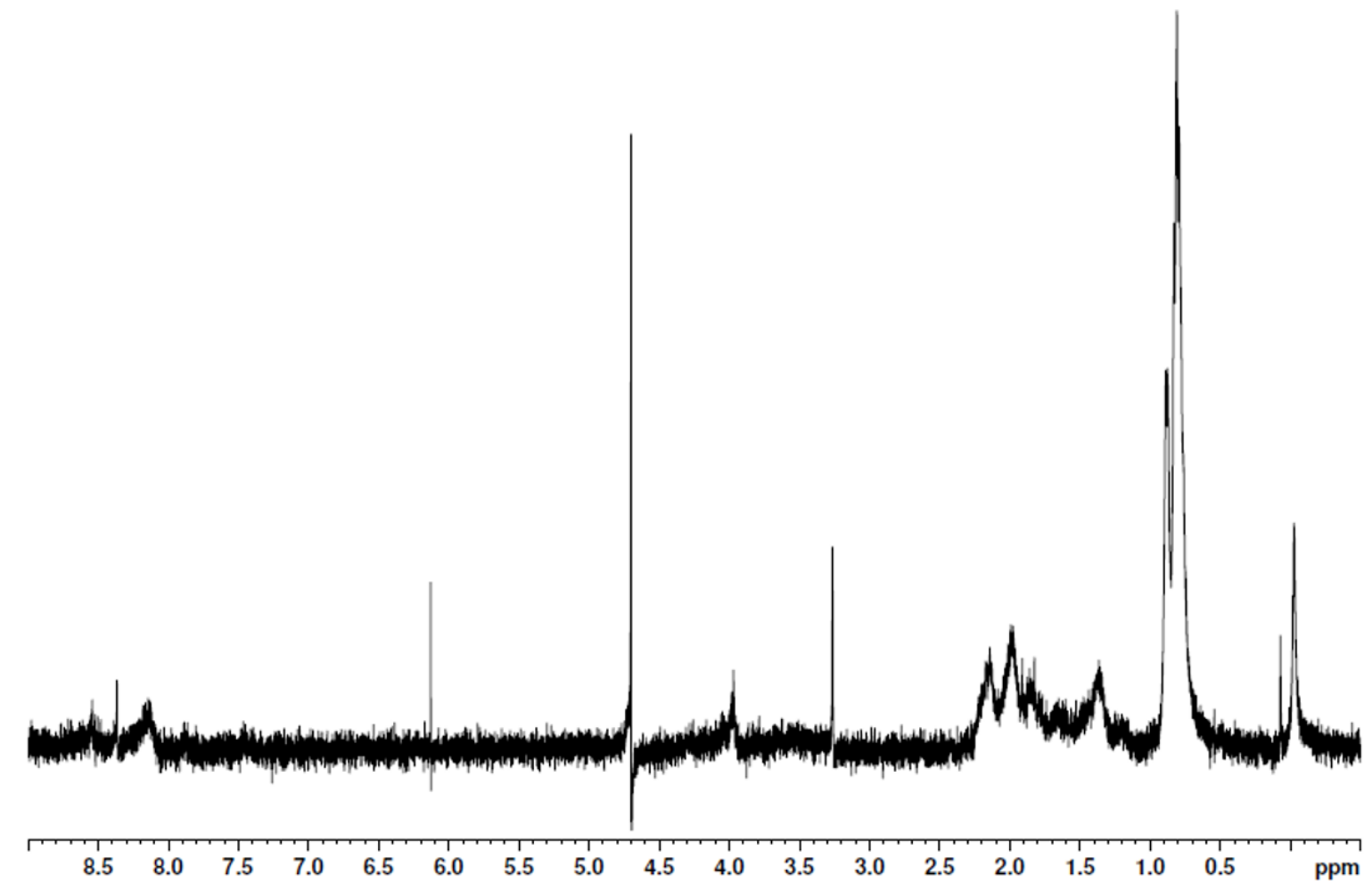

Figure S4. ${ }^{1} \mathrm{H}$ NMR of HO-VEVK(NDI)GG-OT4-GGK(NDI)VEV-OH peptide (DA-3) in $\mathrm{D}_{2} \mathrm{O}$. Spectrum was acquired using a 1 second presaturation pulse to suppress water. Peak broadness presumably due to aggregation of the peptide during the experiment. 


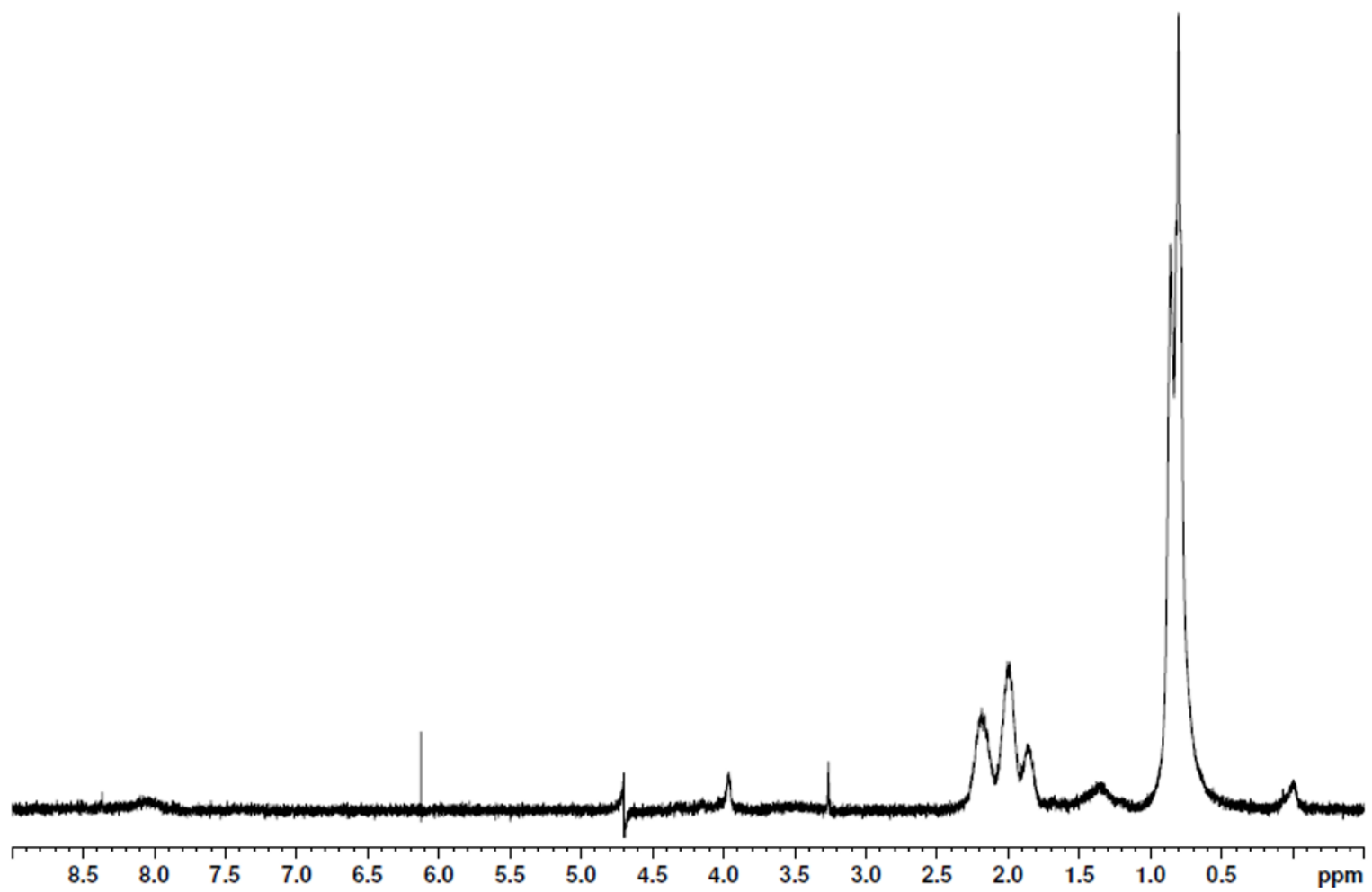

Figure S5. ${ }^{1} \mathrm{H}$ NMR of HO-VEVGK(NDI)G-OT4-GK(NDI)GVEV-OH peptide (DA-2) in $\mathrm{D}_{2} \mathrm{O}$. Spectrum was acquired using a 1 second presaturation pulse to suppress water. Peak broadness presumably due to aggregation of the peptide during the experiment. 


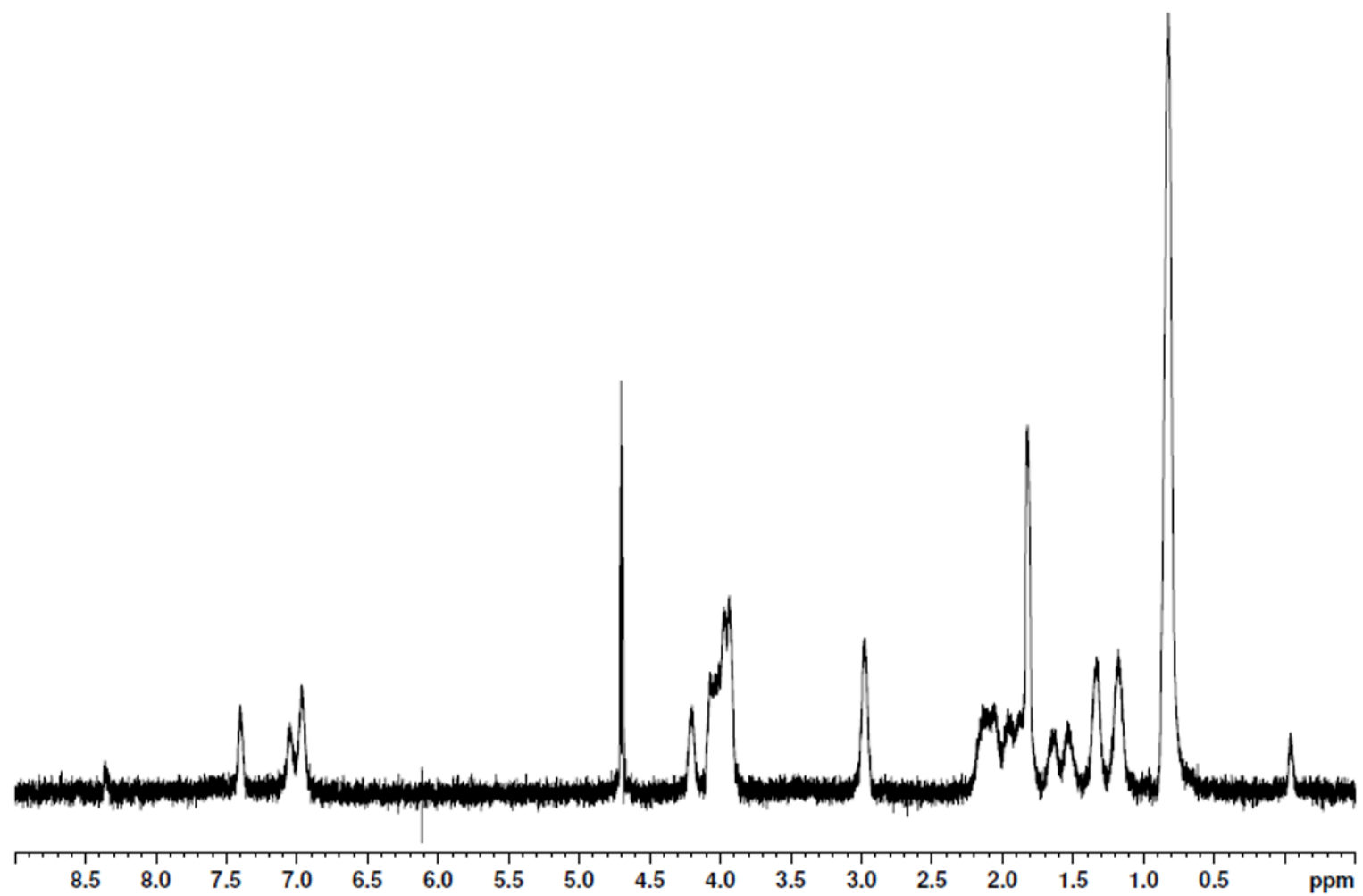

Figure S6. ${ }^{1} \mathrm{H}$ NMR of HO-K(Ac)VEVGG-OT4-GGVEVK(Ac)-OH peptide (C-6) in $\mathrm{D}_{2} \mathrm{O}$.

Spectrum was acquired using a 1 second presaturation pulse to suppress water. Peak broadness presumably due to aggregation of the peptide during the experiment. 


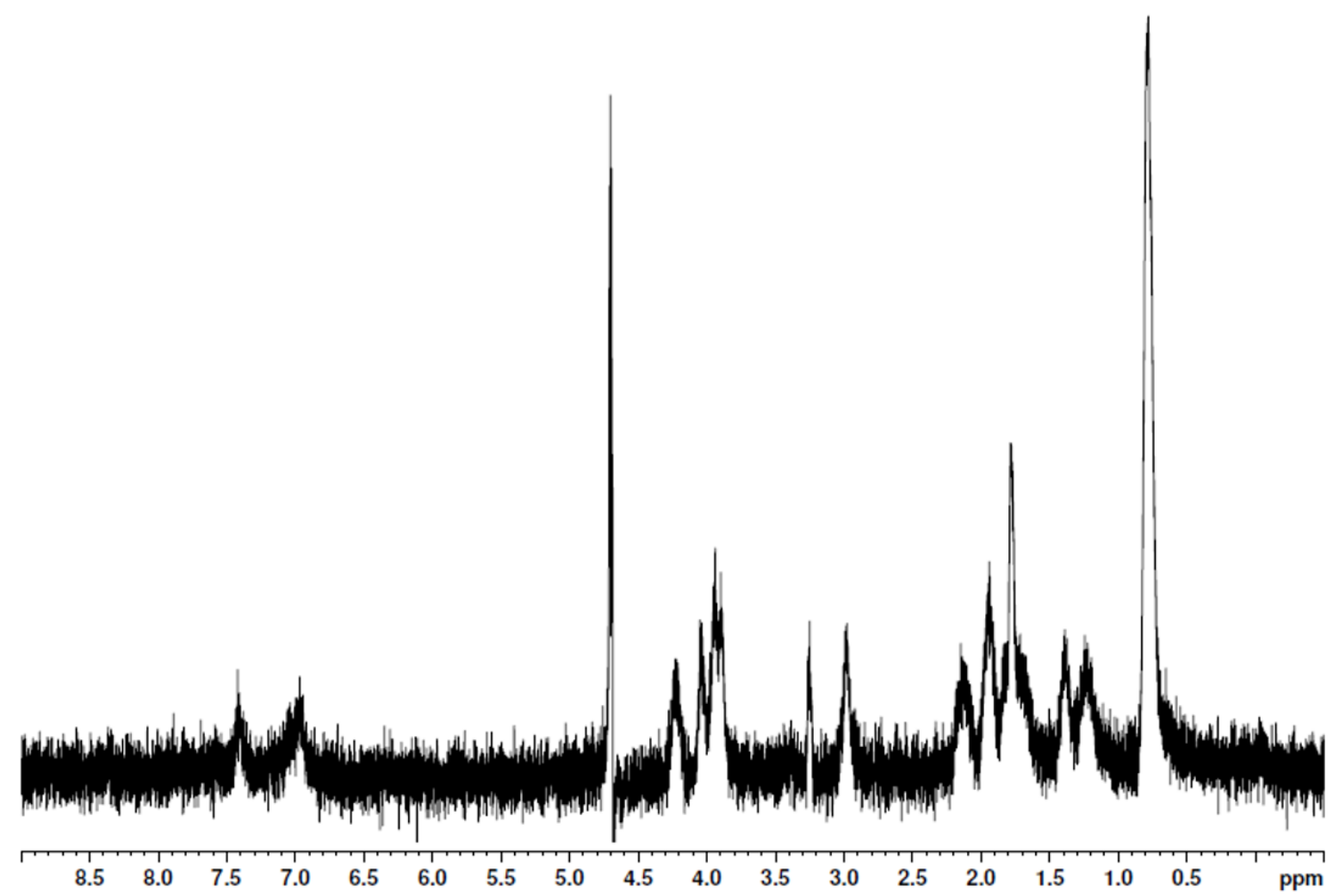

Figure S7. ${ }^{1} \mathrm{H}$ NMR of HO-VEVK(Ac)GG-OT4-GGK(Ac)VEV-OH peptide (C-3) in $\mathrm{D}_{2} \mathrm{O}$.

Spectrum was acquired using a 1 second presaturation pulse to suppress water. Peak broadness presumably due to aggregation of the peptide during the experiment. 


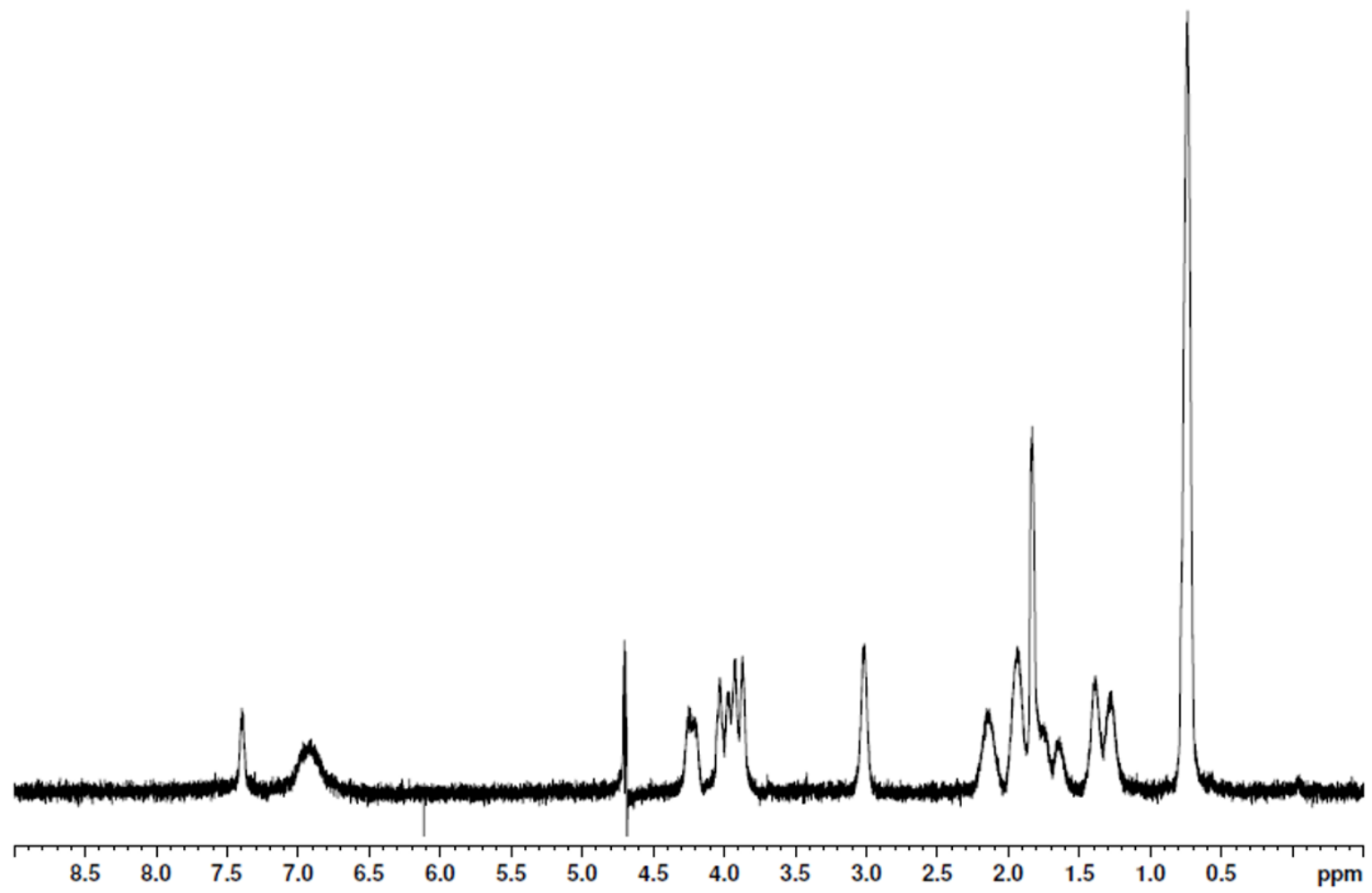

Figure S8. ${ }^{1} \mathrm{H}$ NMR of HO-VEVGK(Ac)G-OT4-GK(Ac)GVEV-OH peptide (C-2) in $\mathrm{D}_{2} \mathrm{O}$.

Spectrum was acquired using a 1 second presaturation pulse to suppress water. Peak broadness presumably due to aggregation of the peptide during the experiment. 


\section{ESI Mass Spectra}

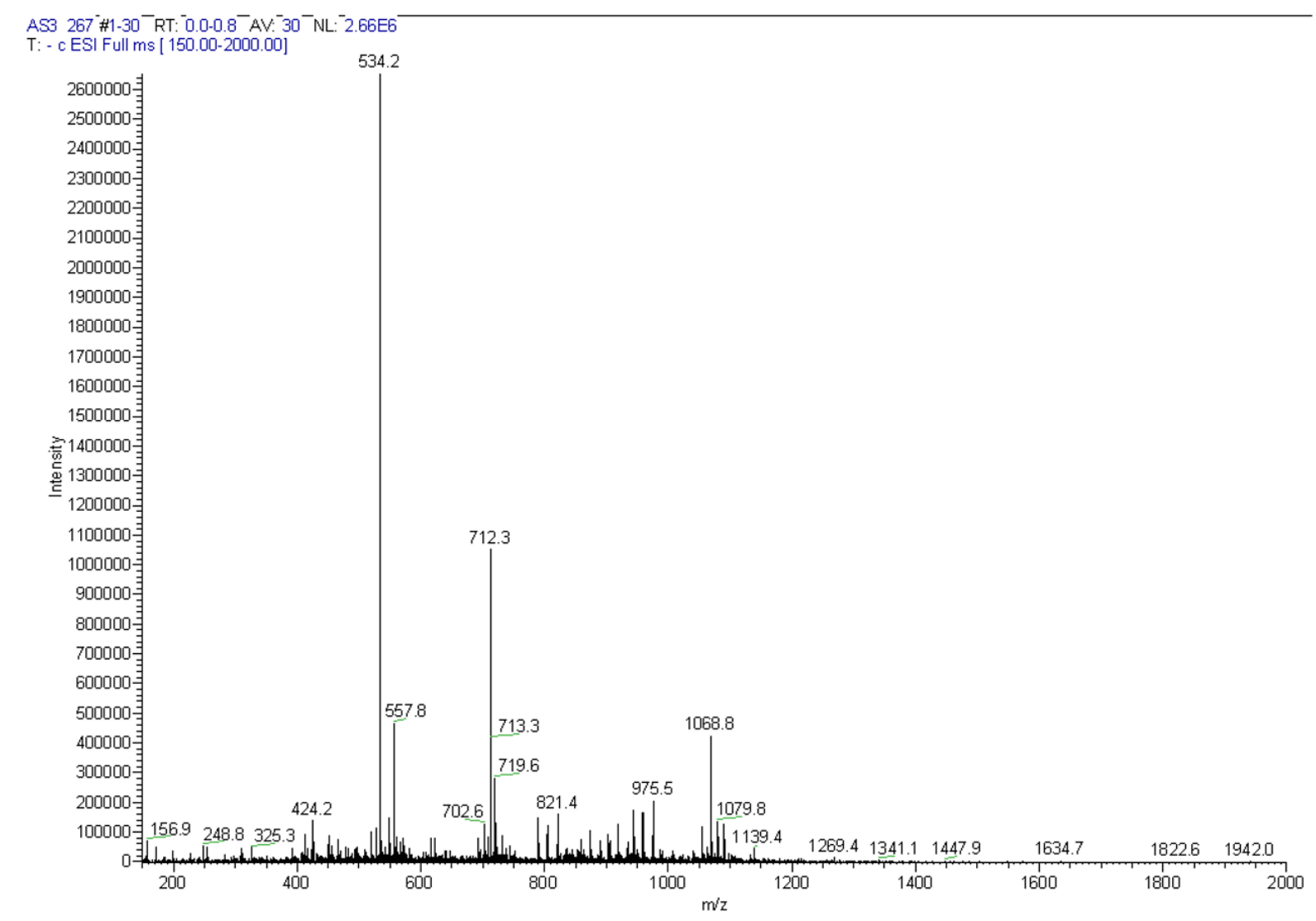

Figure S9. ESI spectrum of HO-K(NDI)VEVGG-OT4-GGVEVK(NDI)-OH peptide (DA-6).

MS (ESI) m/z 1068.8 (M-2H)-2 (calc. 1068.8), m/z 712.3 (M-3H)-3 (calc. 712.2), m/z 534.2 (M-

3H)-3 (calc. 533.9). 


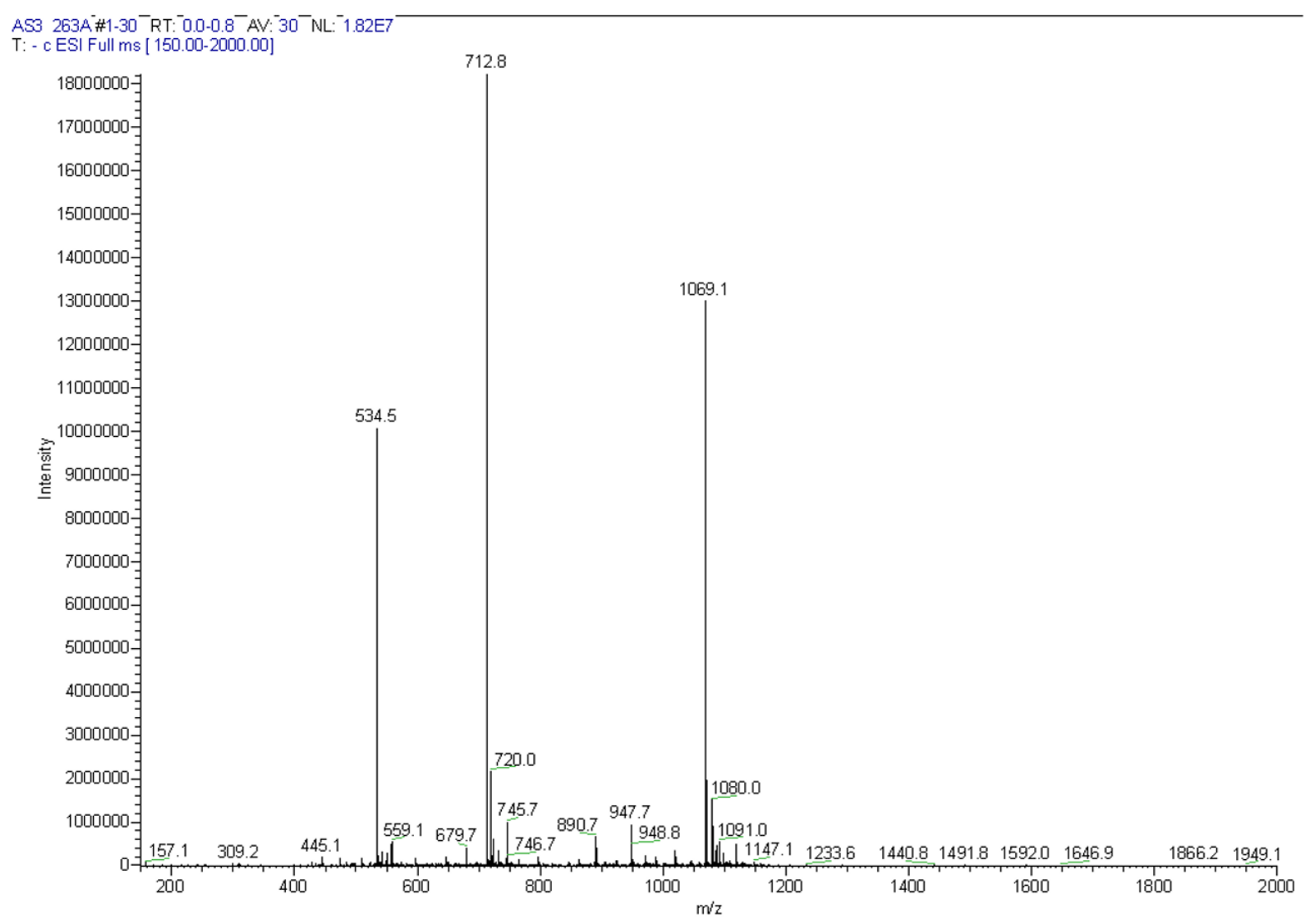

Figure S10. ESI spectrum of HO-VEVK(NDI)GG-OT4-GGK(NDI)VEV-OH peptide (DA-3).

MS (ESI) $m / z$ 1069.1 (M-2H)-2 (calc. 1068.9), m/z 712.8 (M-3H)-3 (calc. 712.4), $m / z 534.5$ (M3H)-3 (calc. 533.9). 


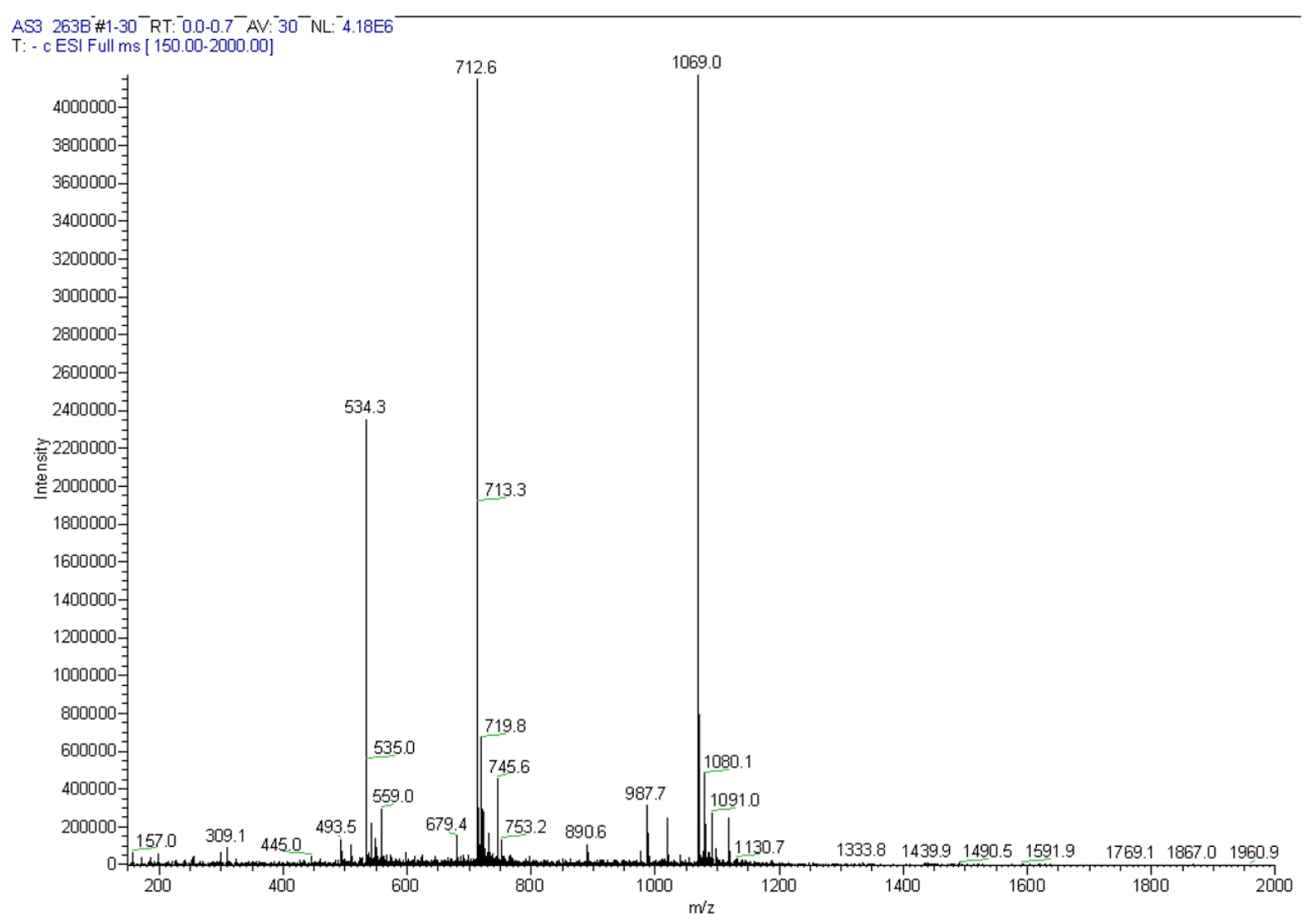

Figure S11. ESI spectrum of HO-VEVGK(NDI)G-OT4-GK(NDI)GVEV-OH peptide (DA-2).

MS (ESI) m/z 1069.0 (M-2H)-2 (calc. 1068.8), m/z 712.6 (M-3H)-3 (calc. 712.2), m/z 534.3 (M3H)-3 (calc. 533.9). 


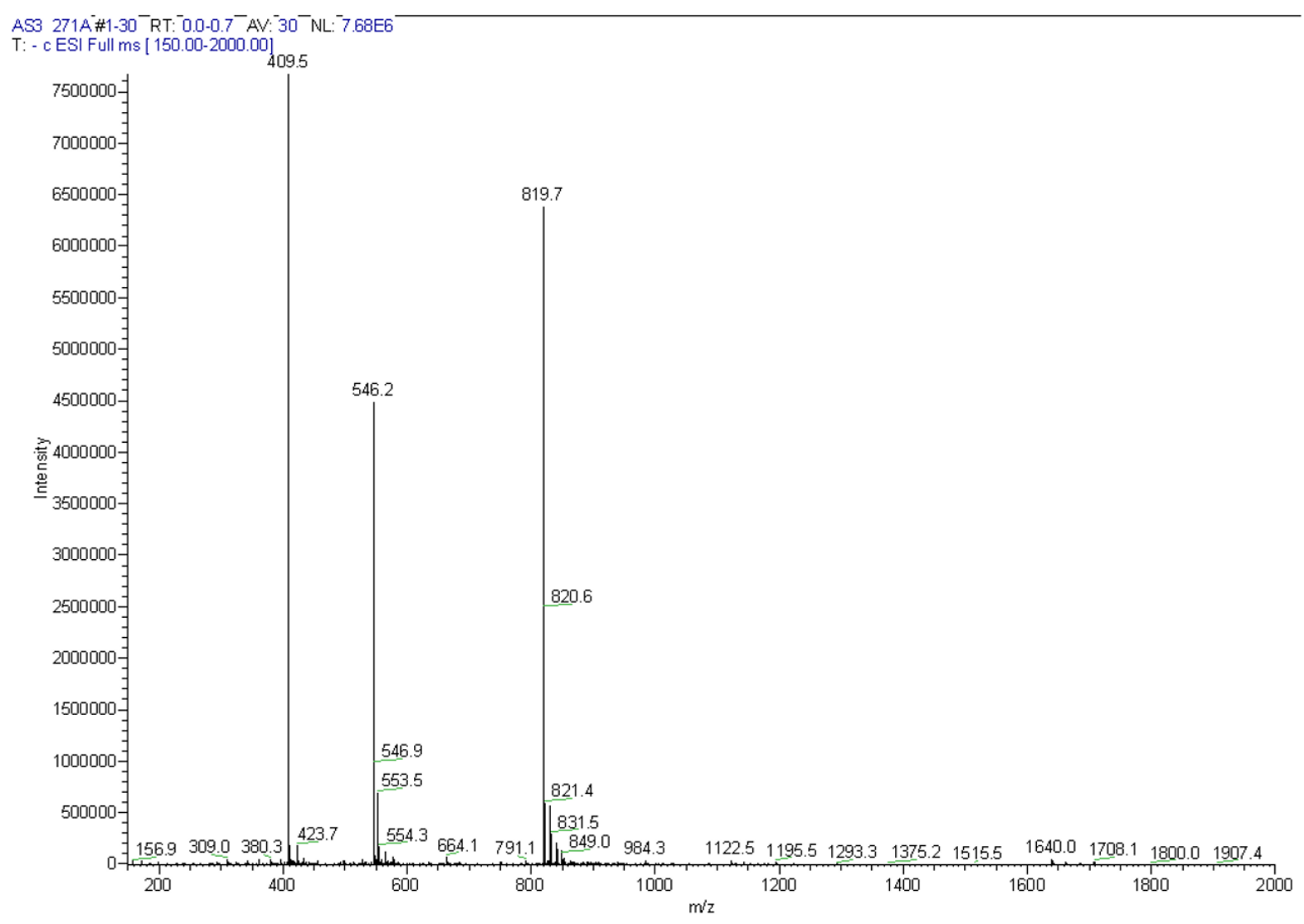

Figure S12. ESI spectrum of HO-K(Ac)VEVGG-OT4-GGVEVK(Ac)-OH peptide (C-6).

MS (ESI) $m / z$ 819.7 (M-2H)-2 (calc. 819.3), m/z 546.2 (M-3H)-3 (calc. 545.9), m/z 409.5 (M-3H)-

3 (calc. 409.1). 


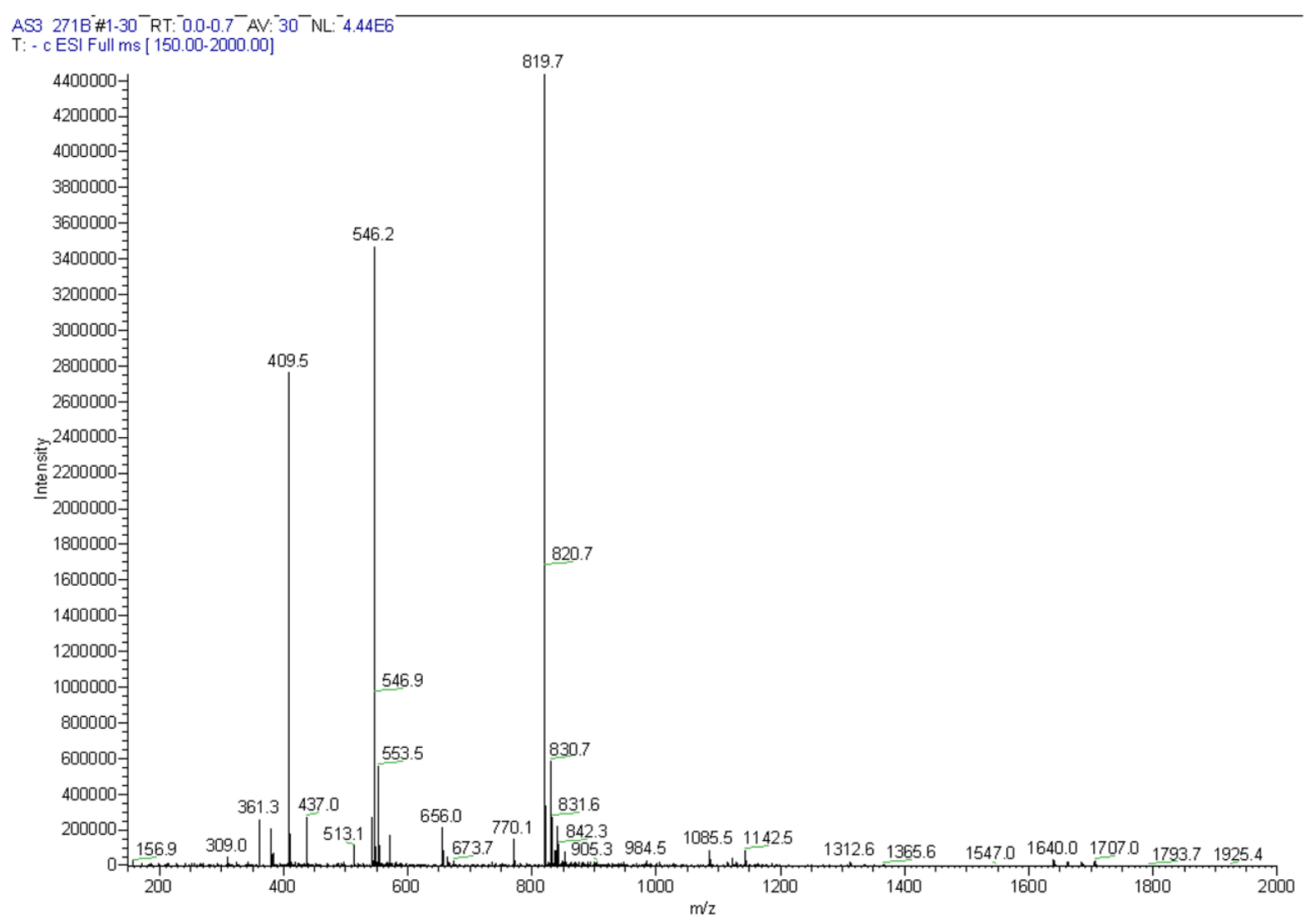

Figure S13. ESI spectrum of HO-VEVK(Ac)GG-OT4-GGK(Ac)VEV-OH peptide (C-3).

MS (ESI) $m / z$ 819.7 (M-2H)-2 (calc. 819.3), m/z 546.2 (M-3H)-3 (calc. 545.9), m/z 409.5 (M-3H)3 (calc. 409.1). 


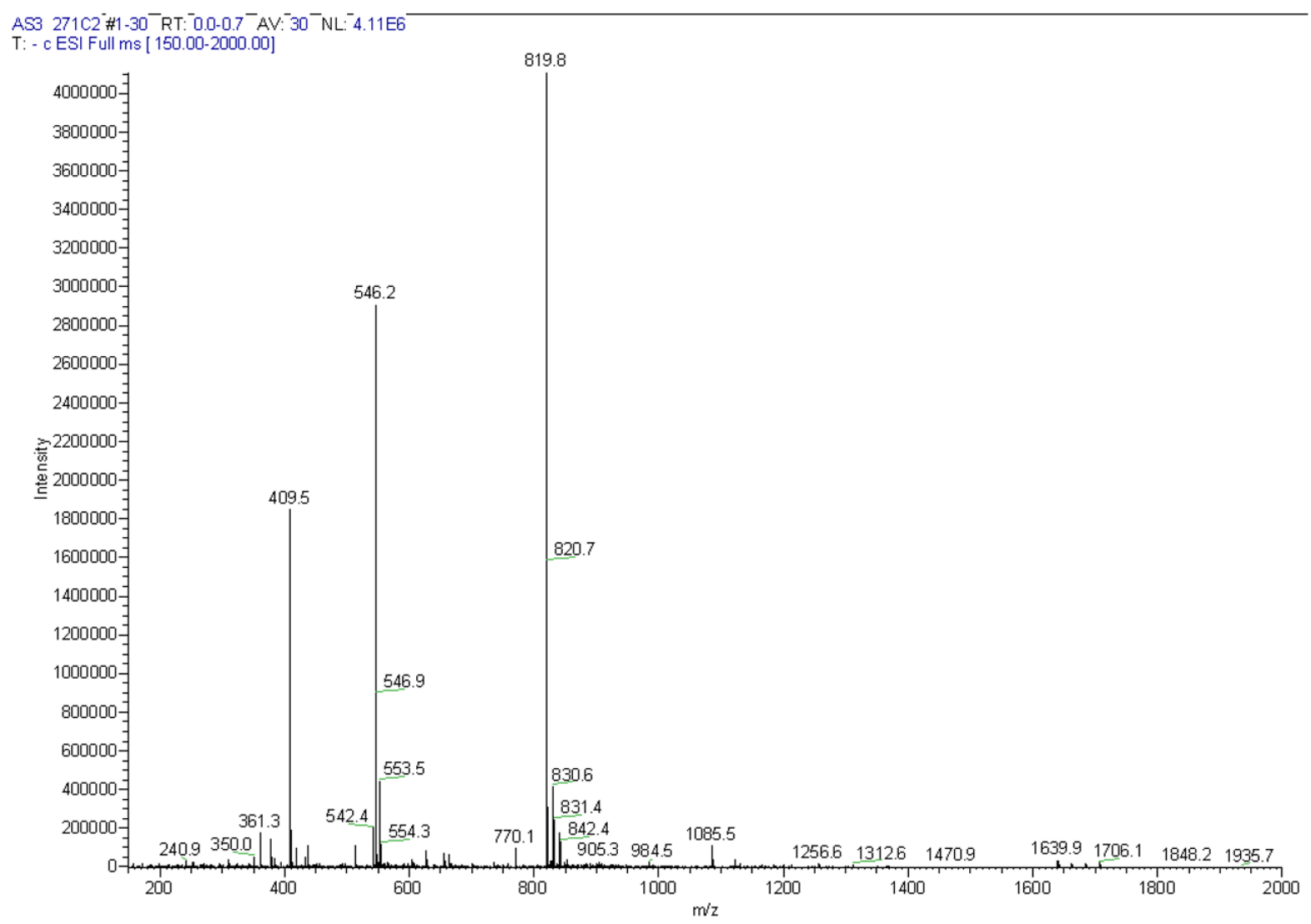

Figure S14. ESI spectrum of HO-VEVGK(Ac)G-OT4-GK(Ac)GVEV-OH peptide (C-2).

MS (ESI) $m / z$ 819.8 (M-2H)-2 (calc. 819.3), m/z 546.2 (M-3H)-3 (calc. 545.9), m/z 409.5 (M-3H)3 (calc. 409.1). 


\section{HPLC Traces of Purified Peptides}

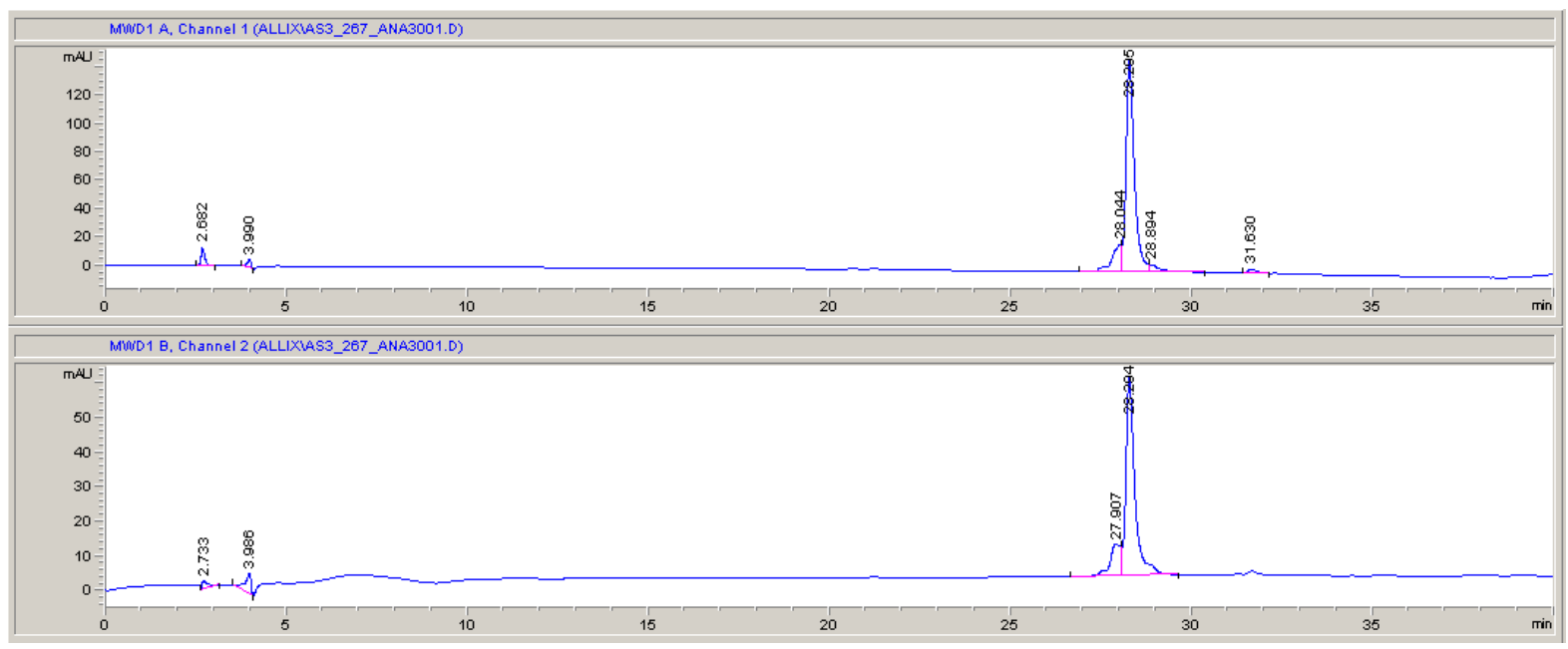

Figure S15. Analytical HPLC trace of purified HO-K(NDI)VEVGG-OT4-GGVEVK(NDI)-OH peptide (DA-6).

Traces monitoring $430 \mathrm{~nm}$ (top) and $260 \mathrm{~nm}$ (bottom). Method: Linear gradient of 11\%-67\% acetonitrile/buffer over 35 minutes, then linear gradient of $67 \%-11 \%$ over 5 minutes.

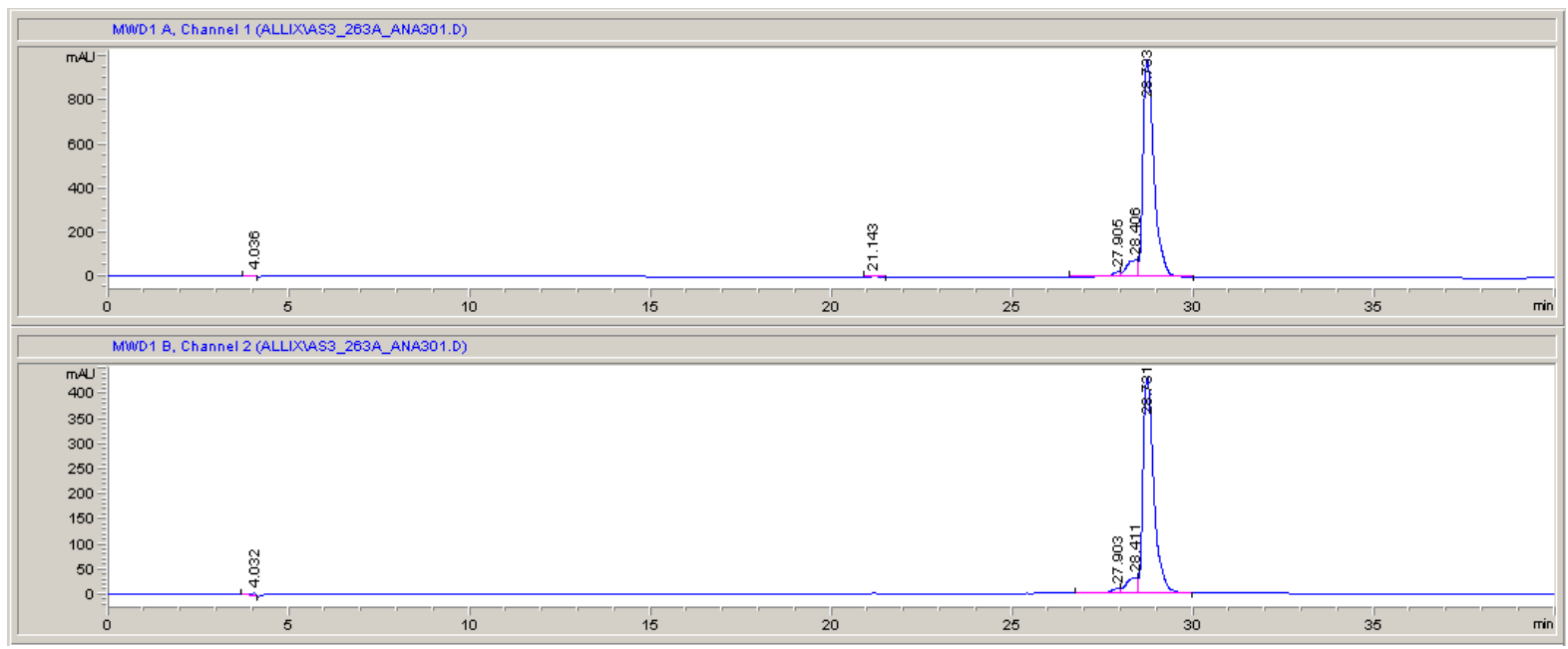

Figure S16. Analytical HPLC trace of purified HO-VEVK(NDI)GG-OT4-GGK(NDI)VEV-OH peptide (DA-3).

Traces monitoring $430 \mathrm{~nm}$ (top) and $260 \mathrm{~nm}$ (bottom). Method: Linear gradient of 11\%-67\% acetonitrile/buffer over 35 minutes, then linear gradient of $67 \%-11 \%$ over 5 minutes. 


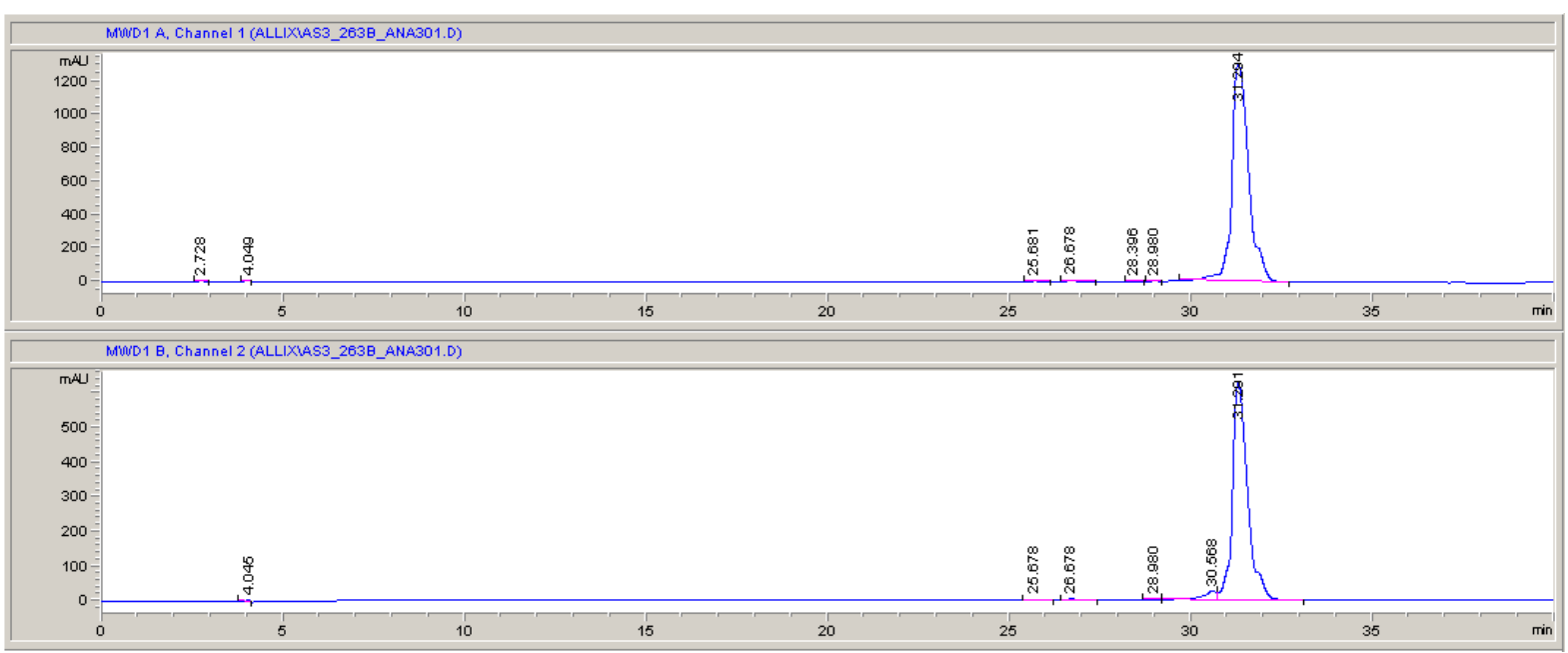

Figure S17. Analytical HPLC trace of purified HO-VEVGK(NDI)G-OT4-GK(NDI)GVEV-OH peptide (DA-2).

Traces monitoring $430 \mathrm{~nm}$ (top) and $260 \mathrm{~nm}$ (bottom). Method: Linear gradient of 11\%-67\% acetonitrile/buffer over 35 minutes, then linear gradient of $67 \%-11 \%$ over 5 minutes.

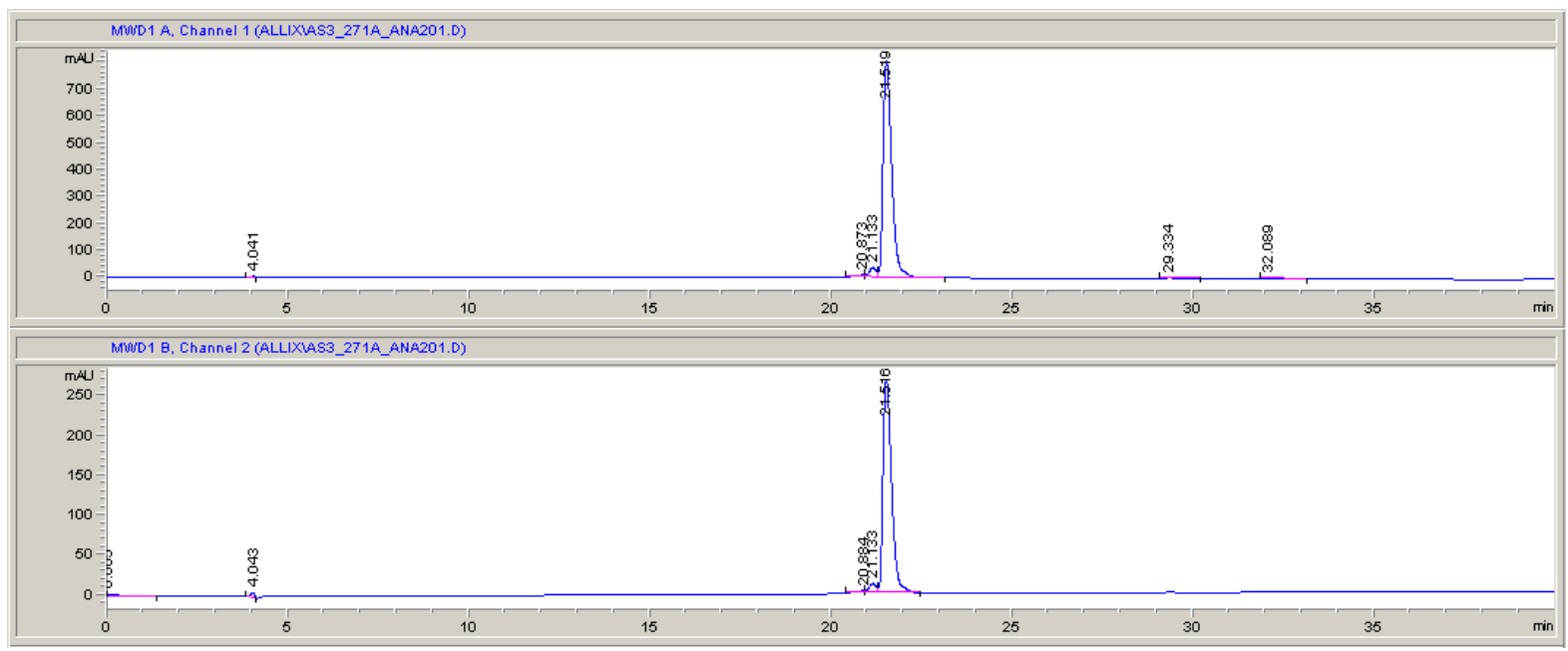

Figure S18. Analytical HPLC trace of purified HO-K(Ac)VEVGG-OT4-GGVEVK(Ac)-OH peptide (C-6).

Traces monitoring $430 \mathrm{~nm}$ (top) and $260 \mathrm{~nm}$ (bottom). Method: Linear gradient of 11\%-67\% acetonitrile/buffer over 35 minutes, then linear gradient of $67 \%-11 \%$ over 5 minutes. 


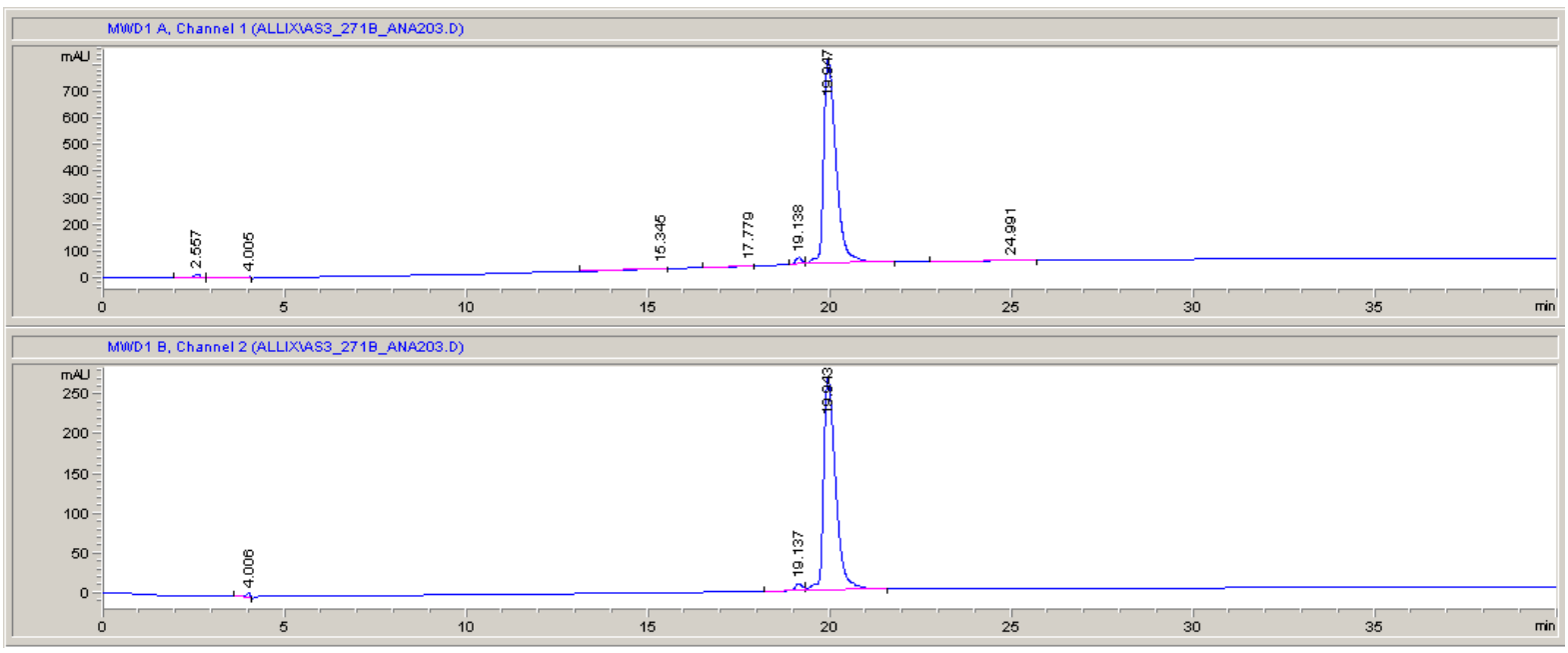

Figure S19. Analytical HPLC trace of purified HO-VEVK(Ac)GG-OT4-GGK(Ac)VEV-OH peptide (C-3).

Traces monitoring $430 \mathrm{~nm}$ (top) and $260 \mathrm{~nm}$ (bottom). Method: Linear gradient of 11\%-67\% acetonitrile/buffer over 35 minutes, then linear gradient of $67 \%-11 \%$ over 5 minutes.

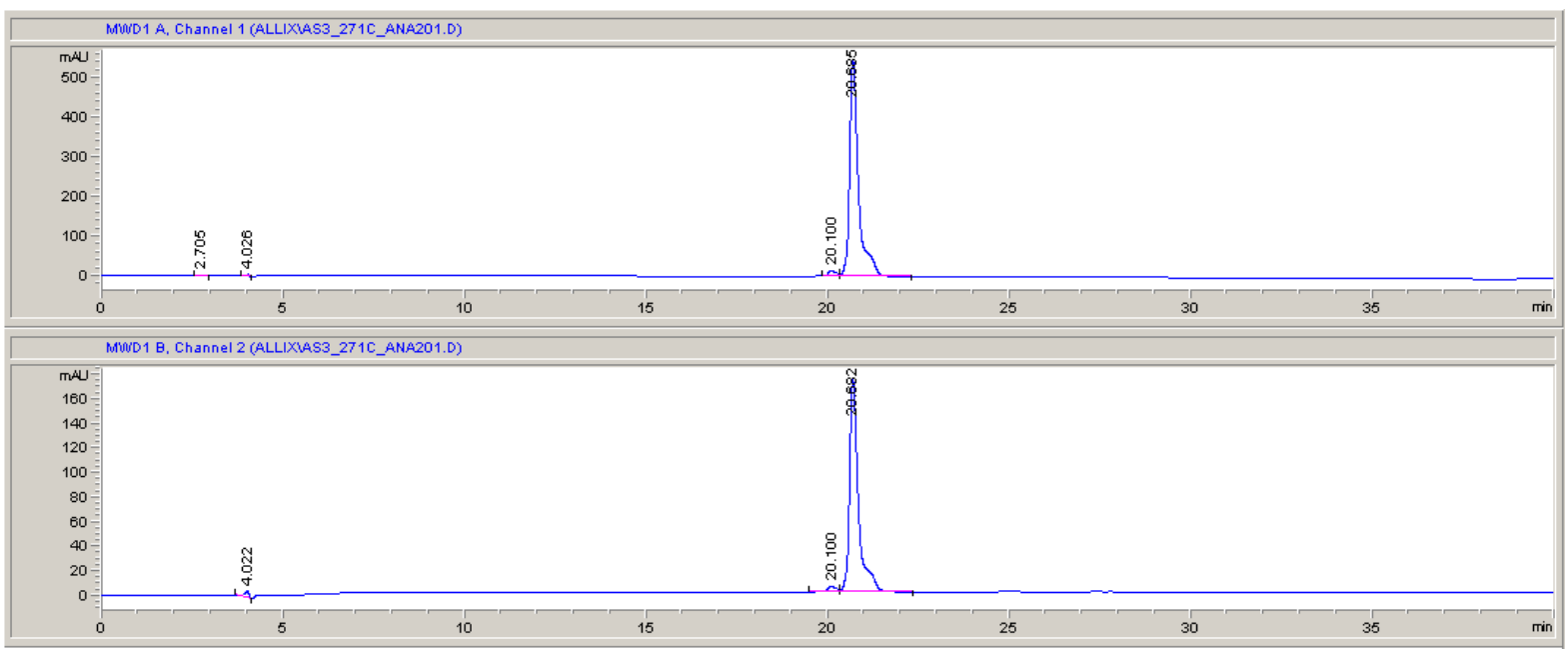

Figure S20. Analytical HPLC trace of purified HO-VEVGK(Ac)G-OT4-GK(Ac)GVEV-OH peptide (C-2).

Traces monitoring $430 \mathrm{~nm}$ (top) and $260 \mathrm{~nm}$ (bottom). Method: Linear gradient of 11\%-67\% acetonitrile/buffer over 35 minutes, then linear gradient of $67 \%-11 \%$ over 5 minutes. 


\section{Additional TEM Images}
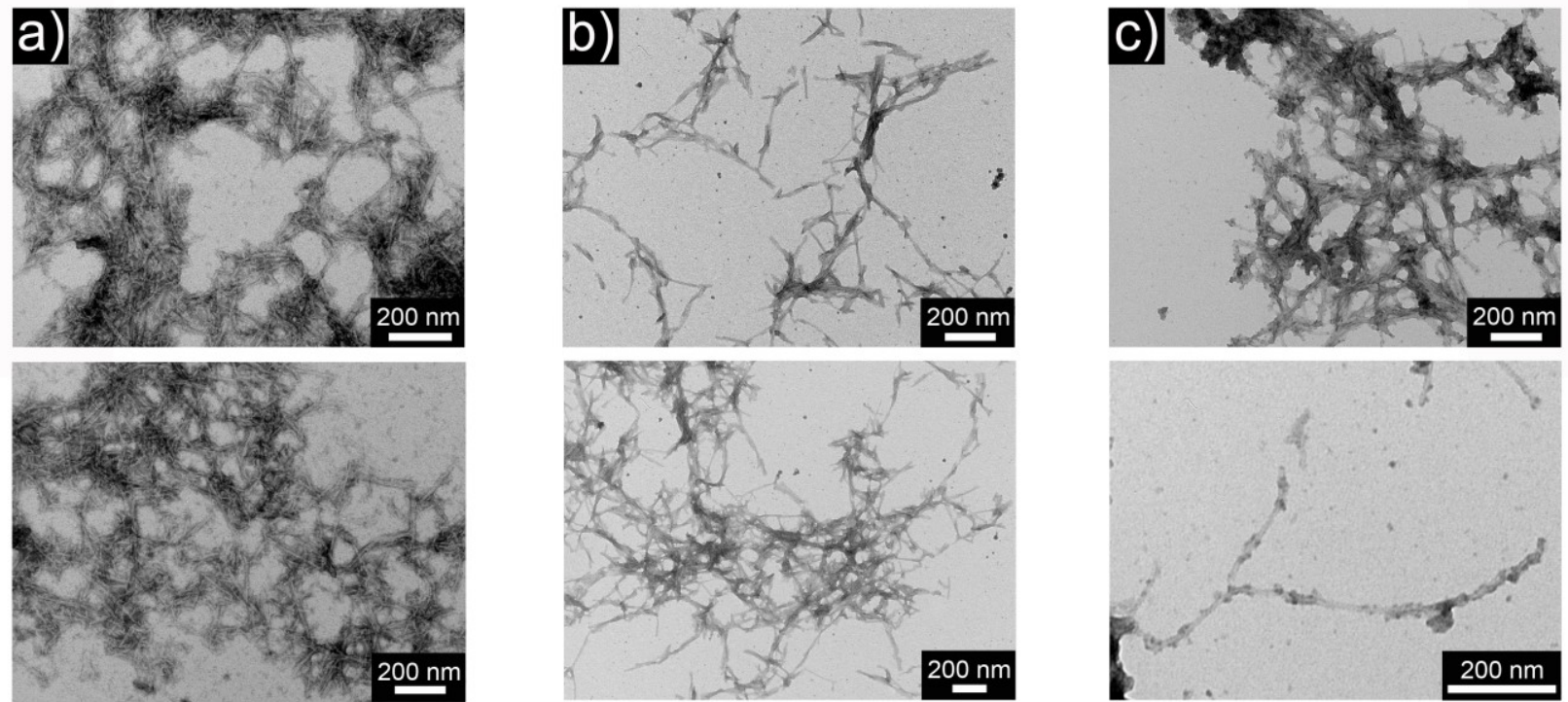

Figure S21. TEM micrographs of acylated control peptides a) C-6, b) C-3, and c) C-2.
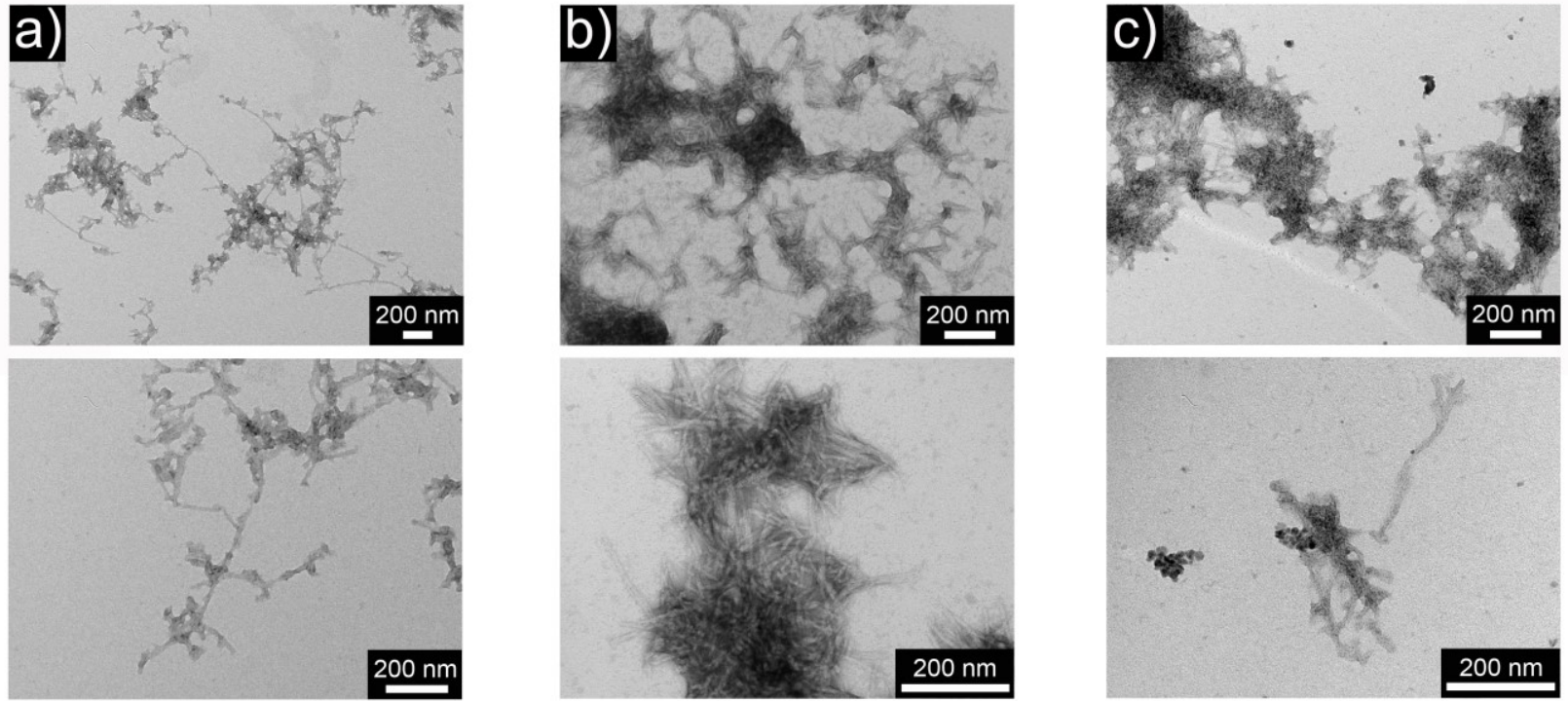

Figure S22. Additional TEM micrographs of donor-acceptor peptides a) DA-6, b) DA-3, and c) DA-2. 

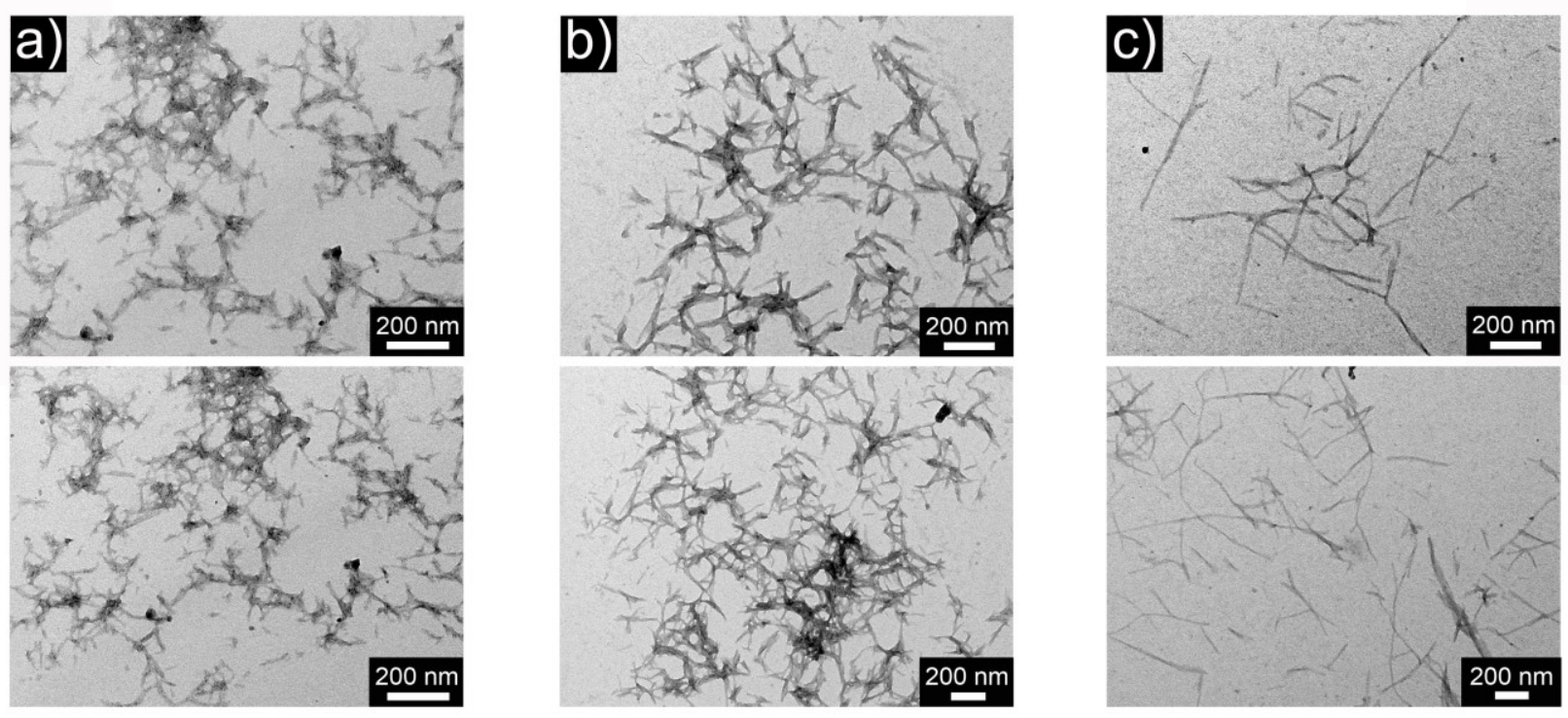

Figure S23. Additional TEM micrographs of 25:75 mixtures of donor-acceptor and control peptides a) 25:75 DA-6:C-6, b) 25:75 DA-3:C-3, and c) 25:75 DA-2:C-2. 


\section{Additional Circular Dichroism Spectra}
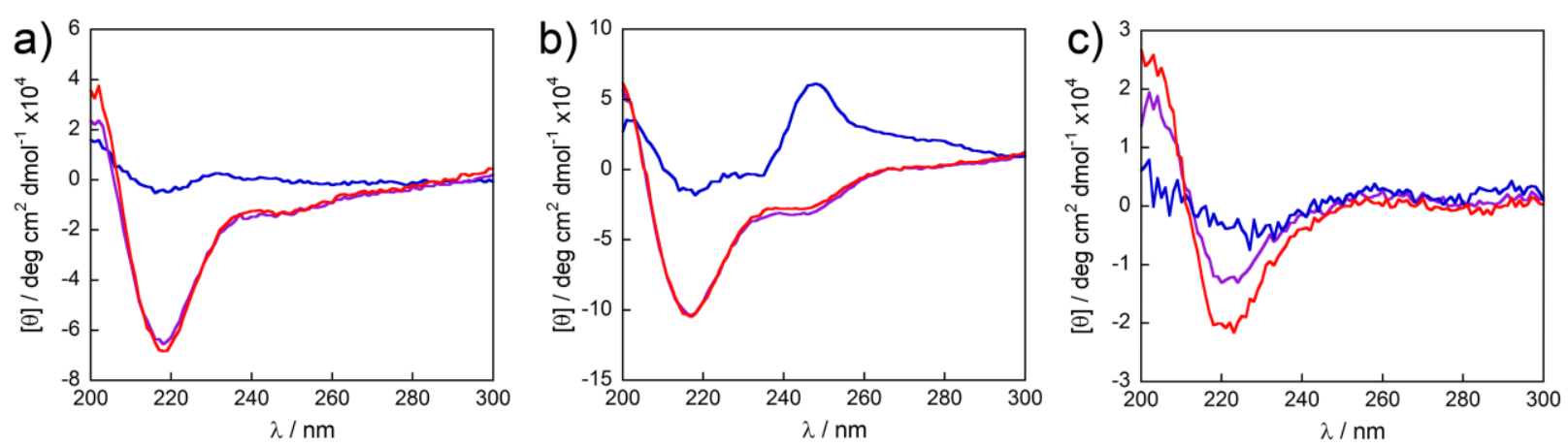

Figure S24. Circular dichroism spectra from 200-300 nm of donor-acceptor (blue lines), control peptides (red lines), and mixtures (10:90 DA:C, purple lines) under assembled conditions (pH 4) at a concentration of $44 \mu \mathrm{M}$. a) DA-6 and C-6, b) DA-3 and C-3, c) DA-2 and C-2. Each peptide displays a minimum at ca. $220 \mathrm{~nm}$, indicative of $\beta$-sheet-like peptide assembly, though this feature is comparatively more intense for the control peptides and mixtures. This may be due to spectral overlap of the imide groups of the NDI acceptors with the peptide amide bonds. 


\section{Additional Ultrafast Spectra and Characterization}

**All measurements were collected using DA-2 unless otherwise noted

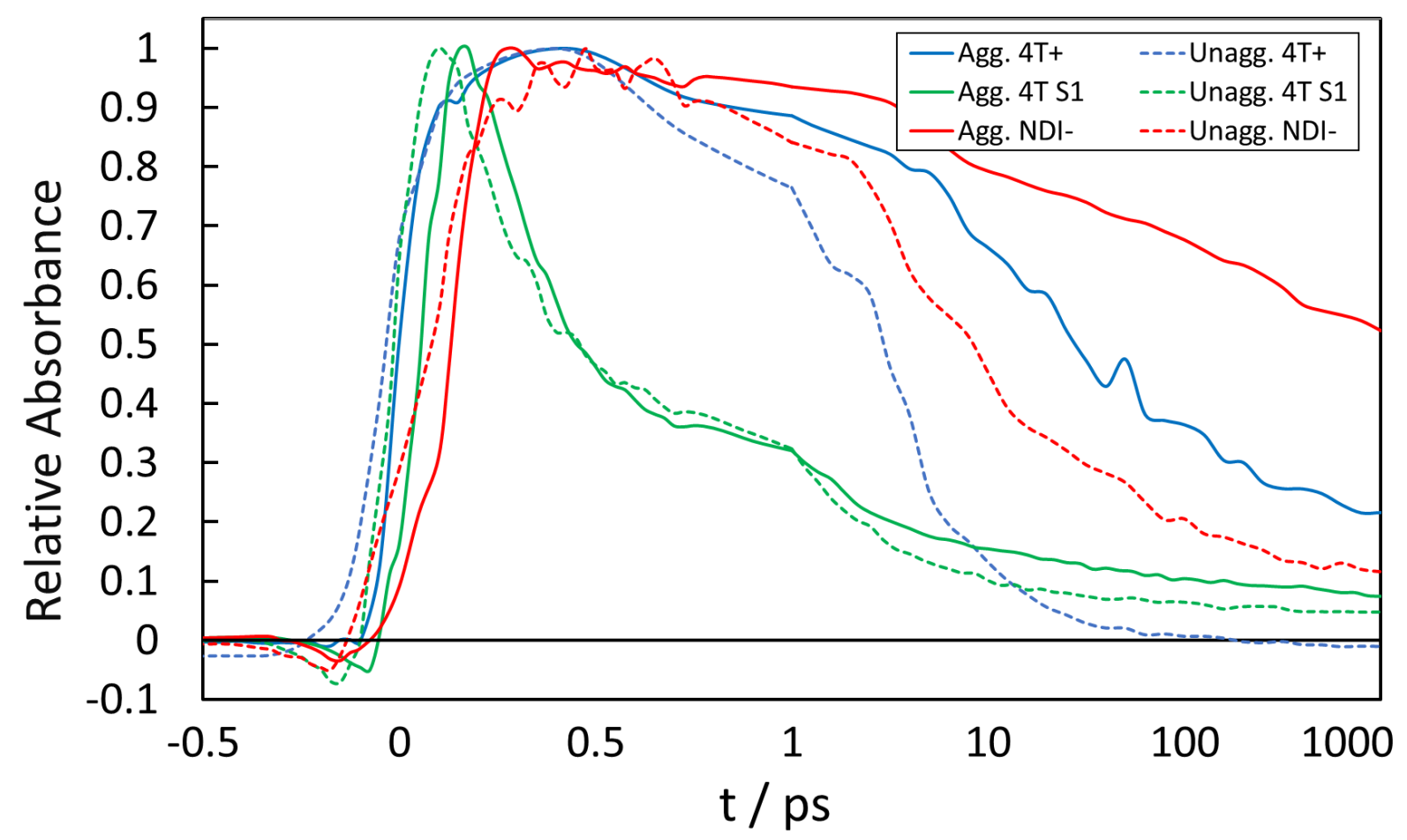

Figure S25. Prototypical transient traces for integrated regions centered about three of the major features of an assembled (solid lines) and unassembled dyad (dashed lines). The OT4 singlet transient (850-900 $\mathrm{nm}$, green) traces show similar kinetics independent of assembly. On the other hand, both the OT4 oxidized polaron (660-700 nm, blue) and reduced NDI (1050-1125 nm, red) exhibit a 1-2 order of magnitude increase in lifetime upon assembly. 
Excitation Energy and Fluence Dependencies. An assembled dyad sample was interrogated to explore excitation energy and fluence dependencies of excited-state dynamics and charge pair formation. Transient spectral signatures were found to be consistent for excitations at different energies along the OT4 visible absorbance band (Figure S26) and over the range of experimentally accessible fluences (Figure S27). By contrast, a significant power dependence was observed for its acylated control, C-2, probed under similar conditions (Figure S28). A linear combination of spectra from these samples was compared with that of a 90:10 mixture (C-2 : DA-2, Figure S29) to elicit evidence of energy migration within the assemblies. 

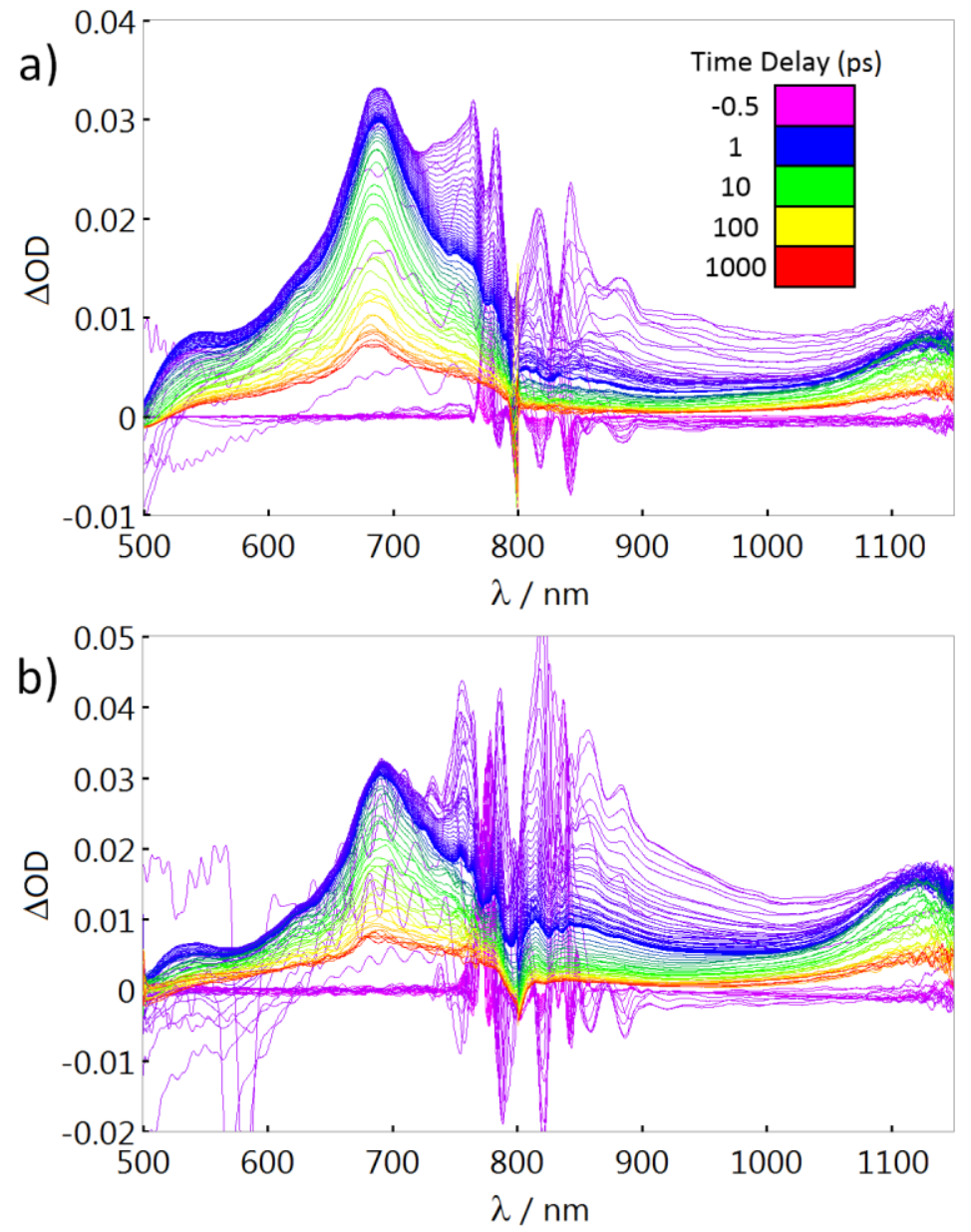

Figure S26. Waterfall plots comparing excitation of DA-2 using 400 (a) and $480 \mathrm{~nm}$ (b) excitation pulses of similar fluence. Both plots exhibit matching spectral features and similar kinetics. Of note, (b) exhibits NIR features with greater intensity than in (a) though some of this may be due to scaling inconsistencies between the 450-800 and 800+ nm segments. 


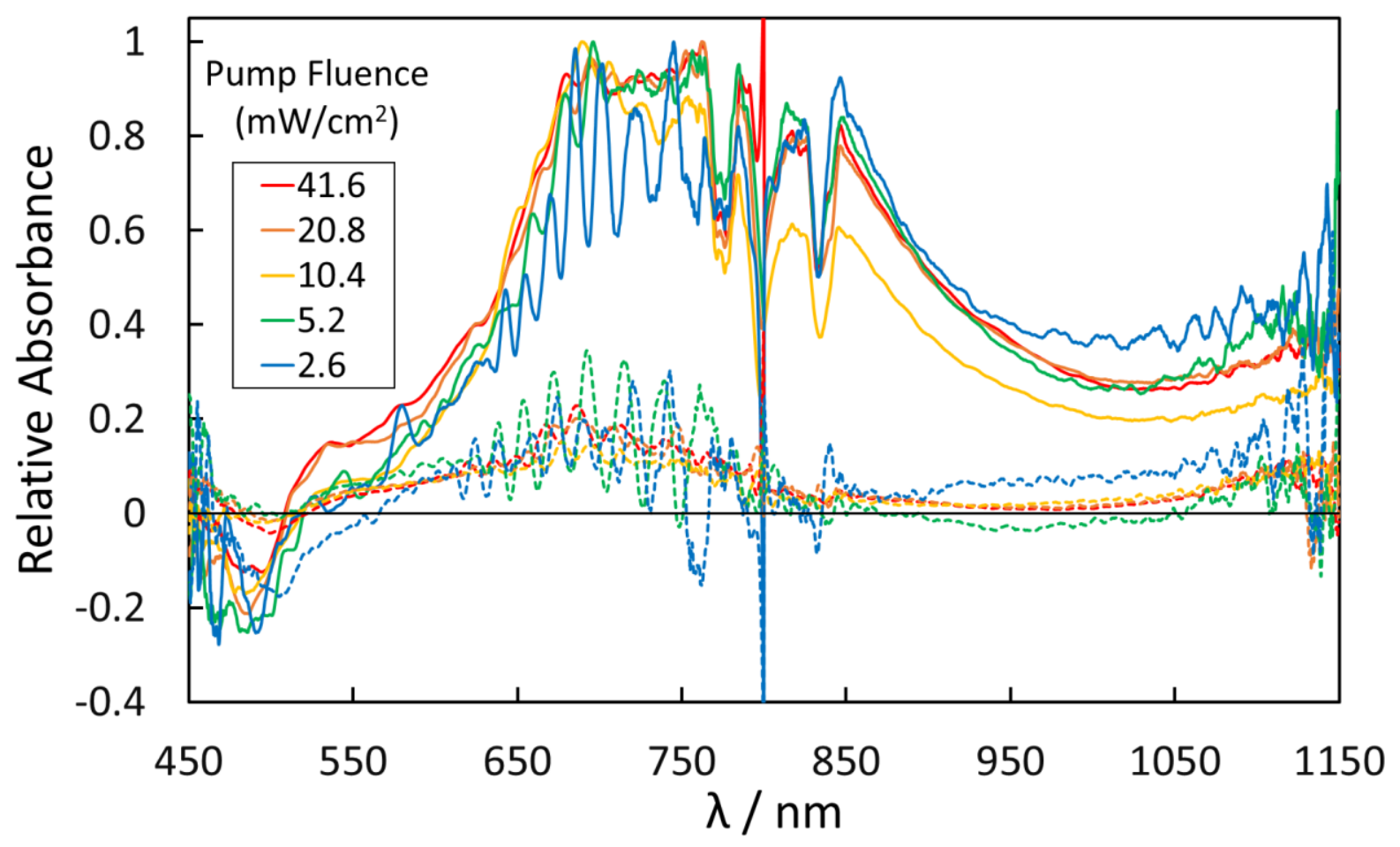

Figure S27. Spectra collected for assembled DA-2 photoexcited at $400 \mathrm{~nm}$ using the indicated excitation fluences. The solid lines represent spectra collected shortly after excitation $(\sim 0.15 \mathrm{ps})$ scaled relative to their peak heights; dashed lines display the spectra remaining $1 \mathrm{~ns}$ after excitation and scaled by the same factors. The similarities in both sets indicate little to no fluence dependence to the dynamics of assembled dyads and the absence of two-photon processes at higher fluences as observed for the acylated control below. 


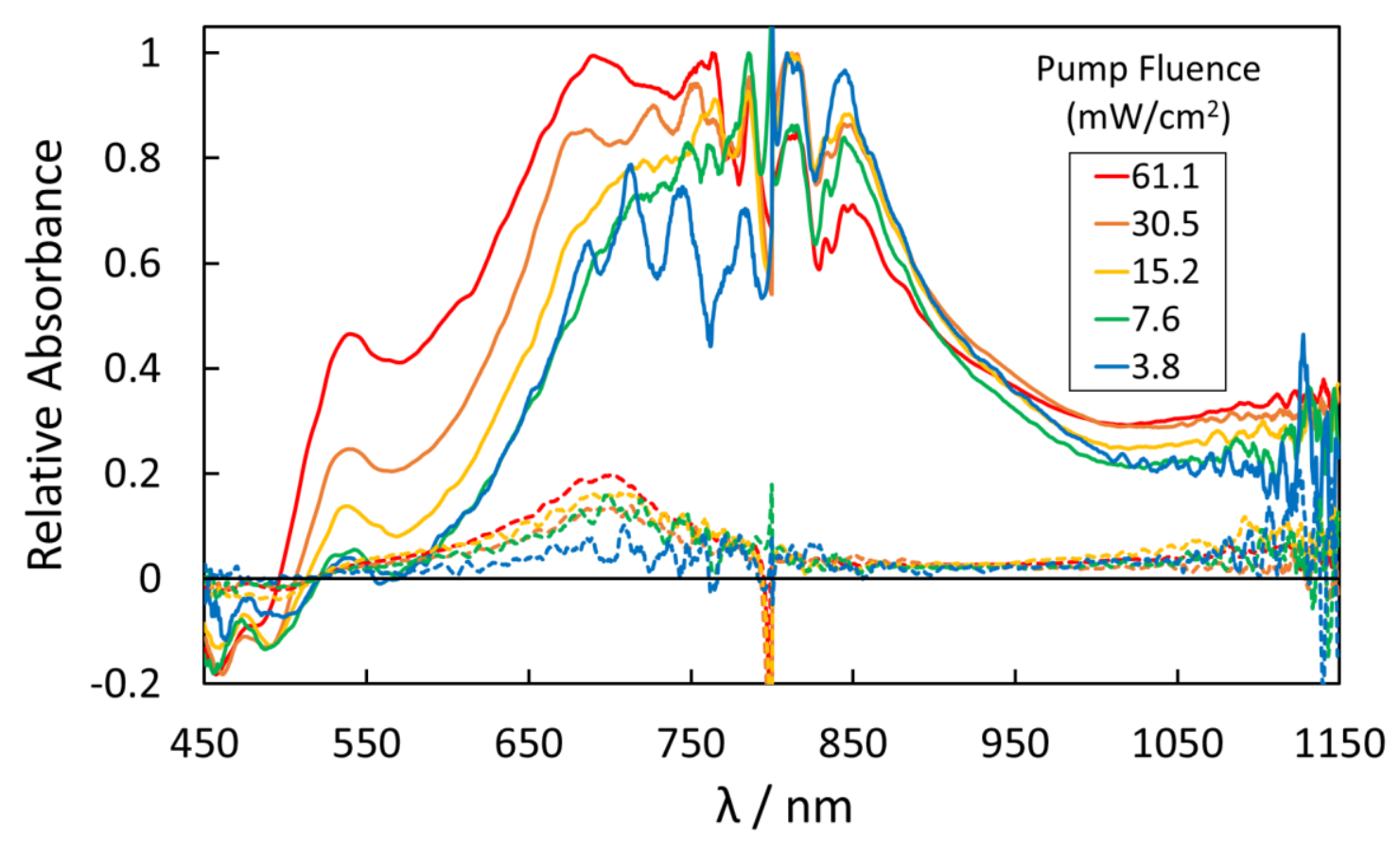

Figure S28. Spectra collected for assembled C-2 photoexcited at $400 \mathrm{~nm}$ using the indicated excitation fluences. The solid and dashed lines have the same designations as in Figure S27; dashed spectra were scaled by a factor of 2 to make their fluence dependence more apparent. At $0.15 \mathrm{ps}$, the $\mathrm{OT}^{+}$signatures at 540 and $680 \mathrm{~nm}$ are most intense at high excitation fluences, while at lower fluences the broad OT4 $\mathrm{S}_{1}$ absorption remains as the only significant feature. Photoinduced formation of $\mathrm{OT}_{4}^{+}$at high fluence excitation is apparent from spectra collected $1 \mathrm{~ns}$ after excitation. 

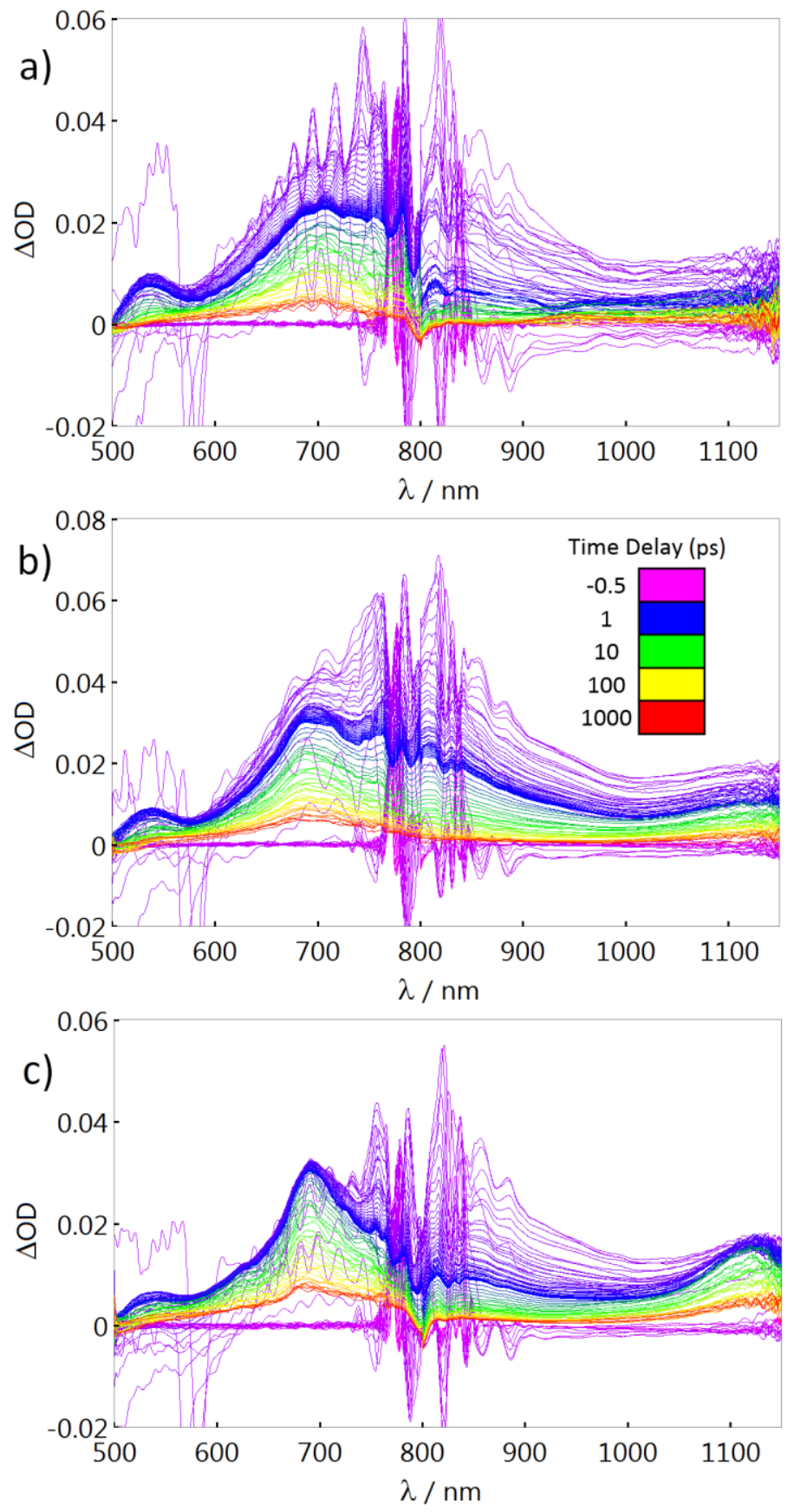

Figure S29. Waterfall plots of transient spectra collected for $400 \mathrm{~nm}$ excitation of C-2 (a), a $90: 10$ C-2 : DA-2 mixture (b) and DA-2 (c) used for the analysis presented within Figure 9 of the text. The integrated signal (1075-1125nm) due to the NDI-less control in (a) is far broader and shorter lived than the signal due to the reduced NDI in (c). The mixture plot (b) depicts that a disproportionately large signal from reduced NDI results with the inclusion of a relatively small added fraction of DA-2 and supports the fluorescence quenching data within the text. 
Spectral dynamics and peptide length. Spectral waterfall plots of transient absorbance data obtained with DA-6 and DA-3 (Figure S30) and DA-2 (Figure 7) illustrate similar spectral dynamics amongst both across assembled and unassembled samples regardless of peptide length. Relative, integrated decay traces from selected regions along the absorbance of these plots (Figure S31) highlight the largely similar kinetics of excited-state decay, charge transfer, and recombination across all three structures unassembled and assembled. The OT4 ${ }^{+}$absorption of DA-2 displays a weak, long-lived component not observed for DA-3 and DA-6 (S28 (e)), which is consistent with the greater self-aggregation of DA-2 in basic solution. Outside of this difference, the time-dependent behavior does not exhibit any noticeable differences with peptide length, supporting similar mechanisms for charge separation and an increased lifetime for charge separation in assemblies. 

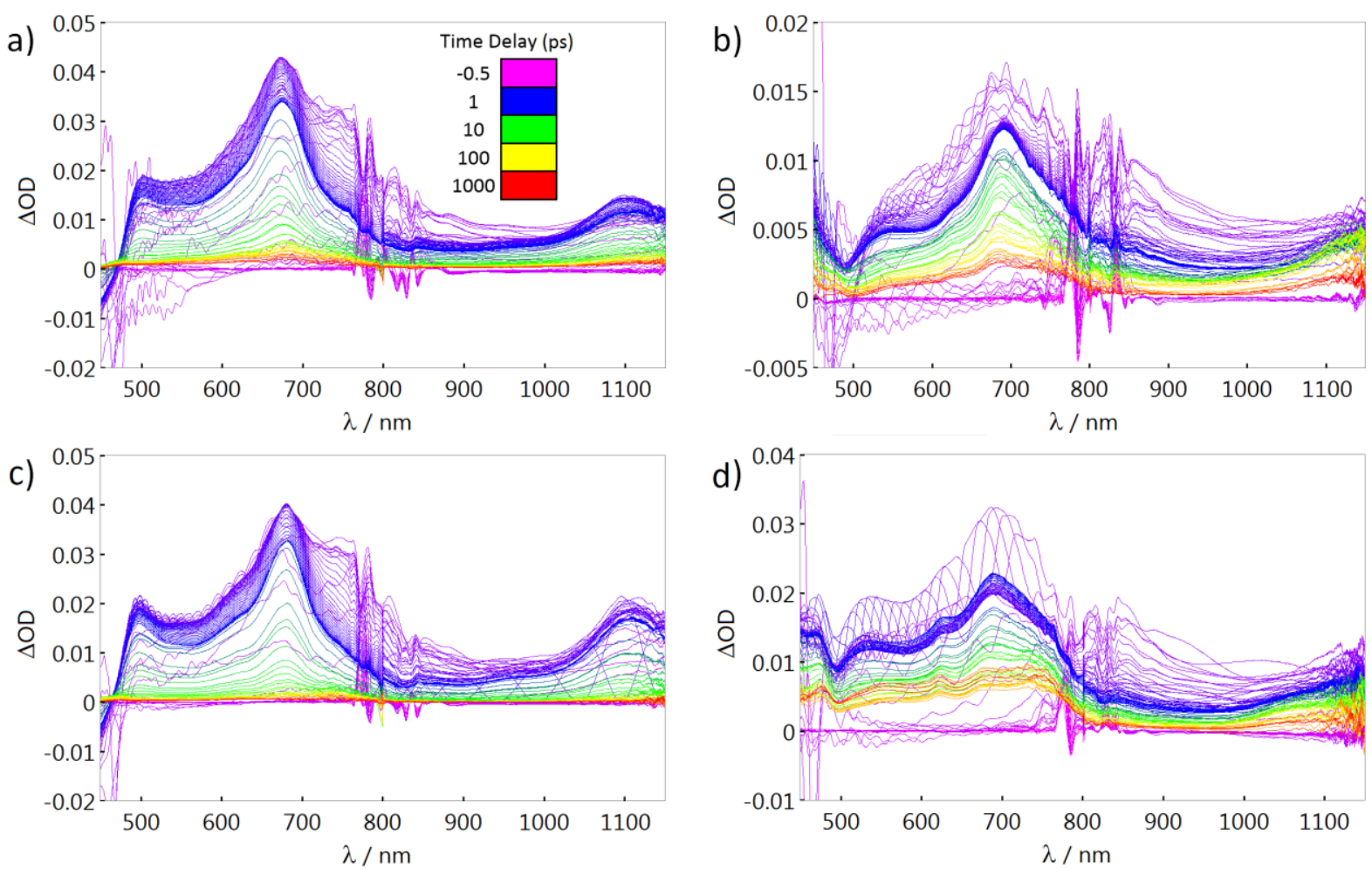

Figure S30. Waterfall plots of transient spectra collected via $400 \mathrm{~nm}$ excitation of unassembled and assembled DA-6 $(\mathrm{a}, \mathrm{b})$ and DA-3 $(\mathrm{c}, \mathrm{d})$, respectively. The spectra resemble those shown in the main text for DA-2. 

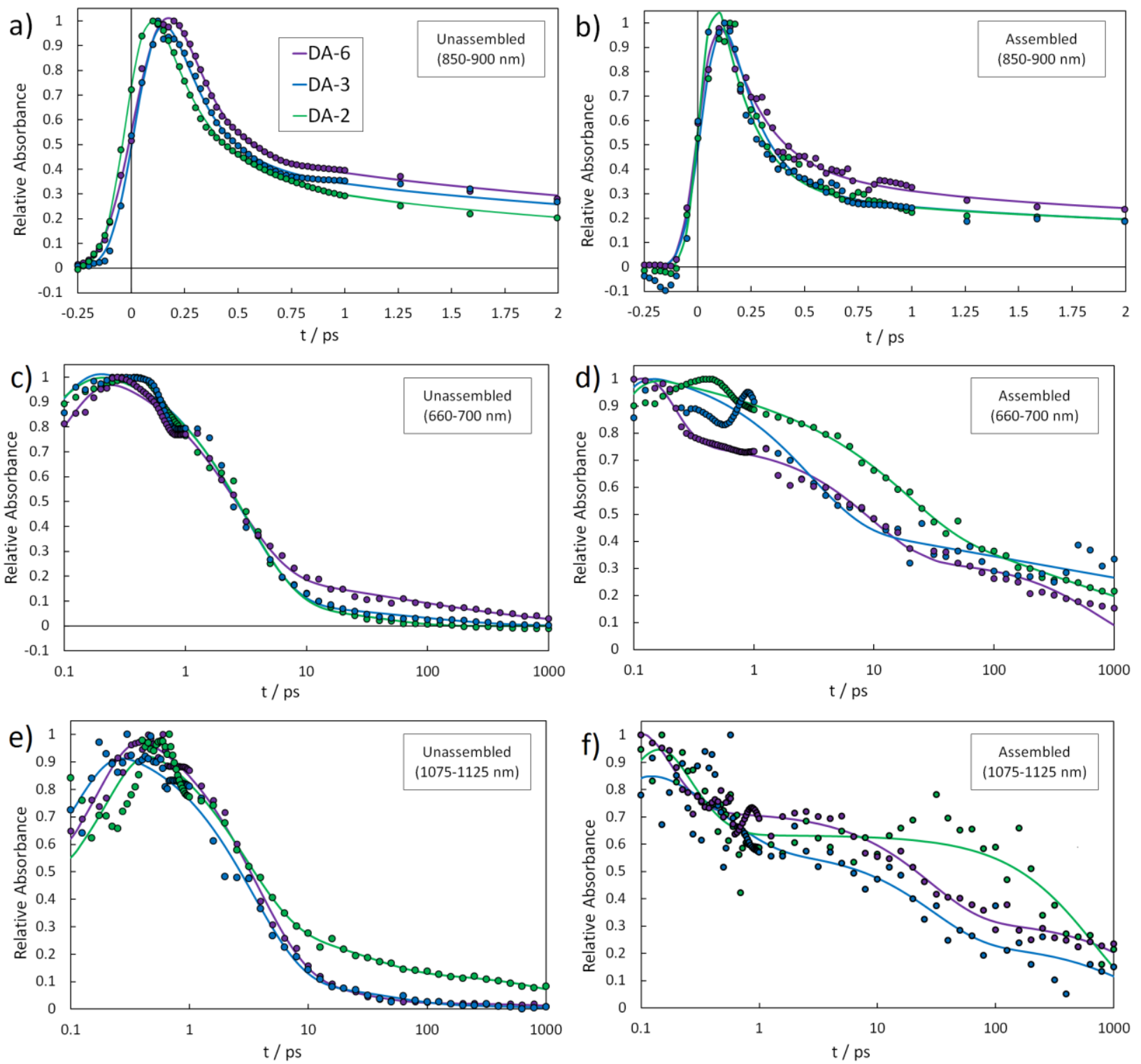

Figure S31. Relative decay traces for different integrated regions of waterfall plots (Figures 7 and S30) obtained with the unassembled and assembled DA-2, DA-3, and DA-6. These three regions largely reflect kinetics of OT4* $(\mathrm{a}, \mathrm{b}), \mathrm{OT}_{4}^{+}(\mathrm{c}, \mathrm{d})$ and $\mathrm{NDI}^{-}(\mathrm{e}, \mathrm{f})$ for the unassembled and assembled dyads, respectively. Plot (f) illustrates the higher stability/longer lifetime of the charged separation at longer delays in assembled structures. These plots demonstrate highly similar time-dependent behaviors for all peptide lengths. 


\section{Spectral Analysis and Fitting Procedures}

Spectral Global Analysis A variation of global analysis (GA) was performed in order to isolate the various components contributing to the progression in Figure 8 of the main text. Ordinarily, global analysis involves applying a loosely defined, pre-conceived kinetic model to tease out components spectrally based on adherence to that model. Validation of the model stems from evaluating how well the component spectra represent a plausible demarcation of the transient populations with reasonable and justifiable spectral features. Instead of making an assumption about the specific kinetic model, the approach utilized here involves limiting the kinetic progressions extracted via singular value decomposition (SVD) to those of populations (positive intensities). Briefly, SVD parses a matrix of data, A, into three other matrices based on Equation S3 below:

$$
A=U S V^{T}
$$

In this case, $\mathrm{U}$ is a matrix that contains information about the components' kinetics, $\mathrm{V}^{\mathrm{T}}$ their spectral profiles, and $\mathrm{S}$ the relative weights of each that will yield linear combinations that reconstruct the original data. The nomenclature $U(n)$, etc. will be used to indicate the $n^{\text {th }}$ row/component of each. Examination of either $\mathrm{U}$ or $\mathrm{V}^{\mathrm{T}}$ without a model often yields negative kinetics, derivative spectral features, and other unassignable or illogical regions and trends. This occurs because SVD delineates the input matrix into the fundamental vectors of the system; these are defined as much by shifting, broadening/narrowing, and exchange between states over time as they are by the absolute spectra of component populations. Thus, the goal here is to linearly combine components of $\mathrm{V}^{\mathrm{T}}$ until non-negative (absolute) kinetics are obtained from $\mathrm{U}$, as an attempt to elicit pure component spectra. 
Examination of Figure 7 of the main text validates an initial assumption that only two significant components contribute to the resulting progressions. $\mathrm{V}^{\mathrm{T}}(1)$ and $\mathrm{V}^{\mathrm{T}}(2)$ give spectra that are clearly contaminated by one another due to their spectral overlap and the presence of timescales on which both populations are significantly changing. This causes an over-representation of component 2 within component 1 , leading to $\mathrm{U}(2)$ behaving more as a derivative function than an absolute one and leading to negative intensities at longer times. Since this is not possible for an absolute population, a small fraction of $\mathrm{V}^{\mathrm{T}}(2)$ is linearly combined with $\mathrm{V}^{\mathrm{T}}(1)$ in varying ratios as shown in Equation S4 with $\left|b_{X}\right| \approx 0.05-0.4 . \mathrm{X}$ is then fit to the original data in a region where $\mathrm{V}^{\mathrm{T}}(1)$ is the most intense (preferably only) spectral contributor. SVD can then be reapplied to the residual matrix left after subtracting out this spectrum fitted to each spectrum in $\mathrm{A}\left(\mathrm{c}_{\mathrm{i}} \mathrm{X}\right)$, as shown in Equation S5.

$$
\begin{aligned}
& X=V^{T}(1)+b_{X} V^{T}(2) \\
& \left(A-c_{i} X\right)=U_{X} S_{X} V_{X}^{T}
\end{aligned}
$$

This yields a component, $\mathrm{V}_{\mathrm{X}}{ }^{\mathrm{T}}(1)$, that is nearly identical to $\mathrm{V}^{\mathrm{T}}(2)$. However, its resulting kinetic progression, $\mathrm{UX}_{\mathrm{X}}(1)$, behaves very differently as a function of time compared to $\mathrm{U}(2)$. The scalar $b_{X}$ is selected to be the value with the smallest absolute value that generates a $U_{X}(1)$ progression in which an exponential model fit through it will always be greater than or equal to zero. This procedure yields a two component system with $\mathrm{X}$ and $\mathrm{V}_{\mathrm{X}}^{\mathrm{T}}(1)$ as the spectral progressions and $\mathrm{U}(1)$ and $\mathrm{U}_{\mathrm{X}}(1)$ as the corresponding kinetic traces and could be extended iteratively in some cases for systems with more than two components. A notable limitation to the technique is that the components must be sufficiently isolatable either spectrally or kinetically for adequate separation. Teasing out additional information encoded within the spectra in predicting 
spectral shifts, broadening/narrowing, and transfer between populations is plausible but beyond the scope of this work.

Kinetic Models Principal kinetic components obtained through global analysis were fitted to exponential decay functions convoluted with the temporal instrument response, namely:

$$
\begin{gathered}
S(t)=S \exp \left(-t / \tau_{S 1}\right) \\
C S(t)=f_{C S 1} \exp \left(-t / \tau_{C S 1}\right)+f_{C S 2} \exp \left(-t / \tau_{C S 2}\right)
\end{gathered}
$$

Here $\mathrm{S}(\mathrm{t})$ corresponds with the short-lived $\mathrm{S}_{1}$ state of OT4, whereas CS(t) corresponds with the charge-separated state (OT4+/NDI-). Values of fitting parameters are presented in Table 1 of the main text. The rise in the CS signal for assembled DA-2 was fit with the 270 -fs rise observed from the decay of $\mathrm{S}_{1}$ OT4. 


\section{References}

(1) Sanders, A. M.; Dawidczyk, T. J.; Katz, H. E.; Tovar, J. D. ACS Macro Lett. 2012, 1 (11), $1326-1329$.

(2) Guo, X.; Watson, M. D. Org. Lett. 2008, 10 (23), 5333-5336.

(3) Magnanelli, T. J.; Bragg, A. E. J. Phys. Chem. Lett. 2015, 6, 438-445.

(4) Zhou, J.; Guo, X.; Katz, H. E.; Bragg, A. E. J. Am. Chem. Soc. 2015, 137, 10841-10850.

(5) Shao, H.; Nguyen, T.; Romano, N. C.; Modarelli, D. A.; Parquette, J. R. J. Am. Chem. Soc. 2009, 131 (45), 16374-16376. 